NBER WORKING PAPER SERIES

\author{
DIFFERENTIAL INFORMATION \\ AND DYNAMIC BEHAVIOR OF \\ STOCK TRADING VOLUME
}

\author{
Hua $\mathrm{He}$ \\ Jiang Wang
}

Working Paper No. 5010

\author{
NATIONAL BUREAU OF ECONOMIC RESEARCH \\ 1050 Massachusetts Avenue \\ Cambridge, MA 02138 \\ February 1995
}

The authors thank Franklin Allen, Bruce Grundy, Richard Kihlstrom, Pete Kyle, Roni Michaely, Krishna Ramaswamy, seminar participants at Columbia University, Hong Kong University of Science and Technology, New York University, Northwestern University, University of California at Berkeley, University of Rochester, University of Utah, University of Wisconsin at Madison, Yale University, Nazen Conference, The Conference on Recent Developments in Asset Pricing and Optimal Trading Strategies at Rutgers University for helpful discussions and comments. Special thanks go to the referee and the editor, Chester Spatt, for many helpful suggestions. The support from the Batterymarch Fellowship Program (for Hua $\mathrm{He}$ ), and from the International Financial Services Research Center and the NTU Career Development Assistant Professorship at MIT (for Jiang Wang) is gratefully acknowledged. This paper is part of NBER's research program in Asset Pricing. Any opinions expressed are those of the authors and not those of the National Bureau of Economic Research.

(C) 1995 by Hua He and Jiang Wang. All rights reserved. Short sections of text, not to exceed two paragraphs, may be quoted without explicit permission provided that full credit, including (C) notice, is given to the source. 


\title{
DIFFERENTIAL INFORMATION AND DYNAMIC BEHAVIOR OF STOCK TRADING VOLUME
}

\begin{abstract}
This paper develops a multi-period rational expectations model of stock trading in which investors have differential information concerning the underlying value of the stock. Investors trade competitively in the stock market based on their private information and the information revealed by the market-clearing prices, as well as other public news. We examine how trading volume is related to the information flow in the market and how investors' trading reveals their private information.
\end{abstract}

\section{Hua $\mathrm{He}$}

Department of Economics

University of California

Berkeley, CA 94720
Jiang Wang

Department of Economics

M.I.T.

Cambridge, MA 02139 and NBER 
Our model also leads to interesting results about the relation between trading volume and price volatility. Exogenous information leads to trading and changes the stock price, since both expectations and the uncertainty about the value of the stock change with the new information. Thus, high volume generated by the exogenous information, private or public, is accompanied by high volatility in prices. However, high volume of trading generated by the existing private information is not accompanied by abnormally high volatility in prices. In this case, the trading is mainly due to investors' need to unwind their positions against each other. It does not generate large changes in their expectations or the uncertainty about the value of the stock. ${ }^{1}$

Our results have several empirical implications. It is well known that stock trading volume exhibits high serial correlation. ${ }^{2}$ In a competitive market, trading occurs when investors receive new information, either public or private. When all information is public, clustering in trading implies that arrivals of new information are serially correlated. ${ }^{3}$ When there is private information, however, our model shows that clustering in trading can be generated by independent information arrivals. Several authors [e.g., Clark (1973), and Lamoureux and Lastrapes (1990, 1992)] have tried to link price volatility with the underlying information flow in the market, and have used volume as a measure of the information flow. Our results show that volume may lag behind the information flow when the information is private. Moreover, volume generated by new information is accompanied by significant price changes, while volume generated by existing (private) information is not. ${ }^{4}$

The setting in this paper is the multi-period counterpart of the classical rational expectations model originally developed by Grossman (1976), and subsequently extended by Hellwig (1980) and Diamond and Verrecchia (1981), among others. It is well known that solving intertemporal trading models with differential information often faces the problem of a high dimensional state space. In general, the equilibrium depends on the hierarchy of expectations, including each investor's expectation of the true state of the economy (first-order expectations), the expectation of other investors' expectations, etc. (higher-order expectations). The number of these expectations needed to characterize the equilibrium generally increases with the history of the economy and so does the dimension of the state space. This often makes the solution intractable. ${ }^{5}$ In the limit when the history is infinite-dimensional, one encounters the "infinite regress" problem [see, e.g., Townsend (1983)] where the order of independent expectations and the dimension of the state space become infinite. In the current model, we show that all higher-order expectations can be reduced to the first-order expectations and we are able to derive a solution to the multi-period rational expectations equilibrium.

Recent literature closely related to this paper includes Pfleiderer (1984), Grundy and Mc- 
Nichols (1989), Brown and Jennings (1989), and Kim and Verrecchia (1991a,b). For example, Grundy and McNichols (1989) consider a three-date model similar to ours and analyze its rational expectations equilibrium. The current model can be viewed as an extension of earlier models in two ways. First, it is a general multi-period model; second, it allows more general information flow to the economy. A truly multi-period model is necessary for studying the dynamic behavior of trading volume. ${ }^{6}$ The more general information flow in a dynamic setting allows us to analyze the impact of different types of information on trading volume.

Several authors have studied dynamic trading models in the case of superior private information where some investors are better informed than the others [see, e.g., Wang (1993), Gennotte and Kyle (1991), and Foster and Viswanathan (1993a)]. In particular, Wang (1994) provides a model of intertemporal trading volume with superior private information. The infinite regress problem vanishes in those models due to the assumption that the better informed investors observe everything known to the less informed investors. In the current model, private information takes the form of differential information, where each investor has some information that other investors do not possess.

The paper is organized as follows. We specify the model in Section 1 and solve the equilibrium in Section 2. In Section 3, we discuss the general properties of the equilibrium, and in Section 4 we examine the behavior of prices and volume, especially the relation between information flow and the dynamics of volume. Section 5 concludes. All proofs are provided in the appendix.

\section{The Model}

We consider a multi-period model of stock trading in which investors receive in each period both private and public information concerning the underlying value of the stock. Investors trade competitively in the market based on their information. There also exists noise in the market that prevents the equilibrium price from fully revealing investors' private information. The model is further defined as follows.

\subsection{Investment Opportunities}

There is a riskless asset and a risky asset ("stock") available for trading at dates $1, \cdots, T-1$. The riskless asset is of perfectly elastic supply with the rate of return $r$ being a non-negative constant. For simplicity, we assume $r=0$. Each share of the stock pays a liquidation value of $\Pi+\delta$ at the final date $T$. Shares of the stock are infinitely divisible and are traded competitively in the stock market. Let the equilibrium share price of the stock at date $t$ be $P_{t}$.

The stock is of a given supply which may change over time. Let $\Theta_{t}$ be the number of shares 
available in the market at date $t, t=1, \cdots, T-1, \theta_{t}$ follows an $\mathrm{AR}(1)$ process:

$$
\Theta_{t}=a_{\Theta} \Theta_{t-1}+\epsilon_{\Theta, t}
$$

where $-1 \leq a_{\theta}<1$ and $\epsilon_{\theta, t} \sim \mathcal{N}\left(0, \sigma_{\theta}^{2}\right)$. The assumption of a random supply of the stock is equivalent to the usual noise trading story, i.e., the liquidity traders have inelastic demands of $1-\theta_{t}$ shares of the stock at $t$, leaving the remaining $\theta_{t}$ shares to the market (assuming that the total number of shares is one). Changes in the liquidity traders' demands will then generate noise trading and changes in the shares supplied to the market. When $a_{\theta}=0$, the amount of noise trading is i.i.d. over time, which is likely to happen when the time between two consecutive trading dates is very large. When $a_{\theta} \rightarrow 1$, the incremental changes of noise trading become i.i.d. over time. This is likely to happen when the time between two consecutive trading dates is very small.

\subsection{Investors}

Let $\mathcal{I}$ be the set of investors in the economy. Investor $i \in \mathcal{I}$ maximizes expected utility of the form:

$$
\mathbf{E}\left[-e^{-\lambda W_{T}^{i}} \mid \mathcal{F}_{i}^{i}\right]
$$

where $W_{T}^{i}$ is his consumption at the final date $T, \mathcal{F}_{t}$ his information set at date $t$, and $\lambda$ his Arrow-Pratt risk aversion coefficient. We have assumed that investors only consume at the last date. It can be shown that allowing intermediate consumption is a straightforward extension.

We assume that $\mathcal{I}=\{1,2, \cdots\}$, i. e., the set of natural numbers. (See Pfleiderer (1984) for a similar approach.) This assumption simplifies the equilibrium price function significantly. For the convenience of aggregation, we define a charge space $(\mathcal{I}, \mathfrak{p}(\mathcal{I}), \mu)$ where $\mathfrak{p}(\mathcal{I})$ is the collection of all subsets of $\mathcal{I}$ and $\mu: \mathscr{}(\mathcal{I}) \rightarrow \Re_{+}$is a finitely additive measure with the property that $\mu(A)=\lim _{N \rightarrow \infty} \frac{1}{N} \#(A \cap\{1,2, \cdots, N\}), \forall A \subseteq \mathcal{I}$ for which the limit exists, where \#(·) denotes the number of elements in the set [see Rao and Rao (1983) and Feldman and Gilles (1985)]. Clearly, investors are equally weighted according to the defined measure. The aggregation of the random variable $z^{i}(i \in \mathcal{I}$ ) over $\mathcal{I}$ with respect to $\mu$ is then given by

$$
\int_{i} z^{i} \equiv \int_{i \in I} z^{i} d \mu(i)=\lim _{N \rightarrow \infty} \frac{1}{N} \sum_{i=1}^{N} z^{i}
$$

\subsection{Information structure}

All investors have the same prior about $\Pi, \delta$ and $\theta_{0}$. Assume that the prior distributions are: $\Pi \sim \mathcal{N}\left(0, \sigma_{\Pi}^{2}\right), \delta \sim \mathcal{N}\left(0, \sigma_{\delta}^{2}\right), \theta_{0} \sim \mathcal{N}\left(0, \sigma_{\theta}^{2} /\left(1-a_{\theta}^{2}\right)\right)$, and $\Pi, \delta, \theta_{0}$ are uncorrelated. Note 
that the prior distribution about $\theta_{0}$ is simply the stationary distribution of $\Theta_{\mathfrak{t}}$. This specific prior is assumed merely for the simplicity of exposition below.

At each date $t$, investor $i \in I$ receives a private signal $S_{t}^{i}$ about the first component of the stock's liquidation value $\Pi$ :

$$
S_{t}^{i}=\Pi+\epsilon_{S, t}^{i}
$$

where $\epsilon_{S, t}^{i}$ is the noise in investor $i$ 's signal. For simplicity, we assume that $\epsilon_{S, t}^{i} \sim \mathcal{N}\left(0, \sigma_{S, t}^{2}\right)$ and are $i . i . d$. across investors. In addition to private signals, investors receive a public signal $Y_{t}$ about $I$ :

$$
Y_{t}=\Pi+\epsilon Y_{1}
$$

where $\epsilon_{Y, t} \sim \mathcal{N}\left(0, \sigma_{Y, t}^{2}\right)$ is the i.i.d. noise in the public signal. Also, $P_{t}$ is observable to all investors. Thus, we can write the investors' information set as follows:

$$
\mathcal{F}_{i}=\left\{\mathcal{F}_{0}, P_{\tau}, Y_{\tau}, S_{\tau}^{i}: 1 \leq \tau \leq t\right\}, \quad i \in \mathcal{I}
$$

where $\mathcal{F}_{0}$ represents prior information as given by the prior distributions. Since $\epsilon_{S, t}^{i}$ is i.i.d. across investors, information is symmetrically distributed among investors. ${ }^{7}$

For simplicity, we shall assume that all the shocks to the economy, $\left\{\epsilon_{\theta, t}, \epsilon_{Y, t}, \epsilon_{S, t}^{i}: i \in \mathcal{I}\right\}$, are jointly normal, independent of each other, independent over time, and independent from $\Pi, \delta$ and $\theta_{0}$. As will become clear, extensions to more general correlation structure, except for the correlation between the signals of different investors are quite straightforward. Note that there are two components in the value of the stock, $\Pi$ and $\delta$. Investors are endowed with information about $\Pi$, but no information is available about $\delta$. Moreover, the total amount of private information is sufficient to infer the true value of $\Pi$ in the current model. As the number of trading dates increases, more private information will be revealed by equilibrium prices and the true value of $I$ will eventually be revealed. However, the other component of the liquidating value $\delta$ is never revealed before the terminal date. Thus, the uncertainty about the value of the stock remains till the end of the economy, when $\sigma_{\delta} \neq 0$.

\section{Equilibrium}

In this section, we solve for the equilibrium of the economy defined above.

\subsection{Notation}

For future references, we first introduce some notation. Define 
- $\mathcal{F}_{t}^{c} \equiv$ the common information available to all investors at date $t$

- $\mathcal{F}_{i}^{p, i} \equiv$ the private information available to investor $i$ at date $t$

- $\mathcal{F}_{i} \equiv$ the total information available to investor $i$ at date $t$.

If we introduce the notation $\underline{Z}_{\imath} \equiv\left(Z_{1}, \cdots, Z_{t}\right)$ for any stochastic process $\left\{Z_{t}\right\}$, i.e., $\underline{Z}_{t}$ represents the history of $Z_{t}$ up to and including $t$, then

$$
\mathcal{F}_{t}^{c}=\left\{\mathcal{F}_{0}, \underline{P}_{t}, \underline{Y}_{t}\right\}, \mathcal{F}_{t}^{p, i}=\left\{\underline{S}_{i}^{i}\right\}, \text { and } \mathcal{F}_{t}^{i}=\left\{\mathcal{F}_{0}, \underline{P}_{t}, \underline{Y}_{t}, \underline{S}_{t}^{i}\right\}
$$

Next, for any stochastic process $\left\{Z_{t}\right\}$, we define respectively expectations conditional on $\mathcal{F}_{t}^{c}$, $\mathcal{F}_{i}^{p, i}$ and $\mathcal{F}_{i}$ :

- $\hat{Z}_{t}^{c} \equiv \mathbf{E}\left[Z_{t} \mid \mathcal{F}_{t}^{c}\right]$

- $\hat{Z}_{t}^{p, i} \equiv \mathbf{E}\left[Z_{t} \mid \mathcal{F}_{t}^{p, i}\right]$

- $\hat{Z}_{t}^{i} \equiv \mathbf{E}\left[Z_{t} \mid \mathcal{F}_{t}\right]$.

Finally, let

- $\mathbf{E}_{t}^{[}[\cdot] \equiv \mathbf{E}\left[\cdot \mid \mathcal{F}_{t}^{c}\right], \mathbf{E}_{t}^{p, i}[\cdot] \equiv \mathbf{E}\left[\cdot \mid \mathcal{F}_{t}^{p, i}\right], \mathbf{E}_{t}^{i}[\cdot] \equiv \mathbf{E}\left[\cdot \mid \mathcal{F}_{t}^{i}\right]$

- $\operatorname{Vit} c[\cdot] \equiv \operatorname{Var}\left[\cdot \mid \mathcal{F}_{t}^{c}\right], \operatorname{Ver}_{t}^{p, i}[\cdot] \equiv \operatorname{Var}\left[\cdot \mid \mathcal{F}_{t}^{p, i}\right], \operatorname{Vit}[\cdot] \equiv \operatorname{Var}\left[\cdot \mid \mathcal{F}_{t}^{i}\right]$

denote respectively the expectations and variances conditional on the different information sets defined above. In particular, let

- $o_{t}^{c} \equiv V_{t}^{c}[\Pi]$ and $o_{t} \equiv V_{t}^{i}[\Pi]$

denote respectively the conditional variances of the underlying asset value $\Pi$ for an investor who only observes public information and an investor who observes both public and private information. Given that there is perfect symmetry among investors in the quality of their private information, $V_{i}^{i}[\Pi]$ should be the same for all investors. Thus we omit the superscript $i$ and use $o_{t}$ to denote it.

Under differential information, the state of the economy at date $t$ depends on the whole history of the economy up to and including $t$. Define $\phi_{t}$ as the vector of state variables of the economy. Then, $\Phi_{t}=\left(I ; \underline{\Theta}_{t} \underline{Y}_{t} ;\left\{\underline{S}_{t}^{i}\right\}_{i \in T}\right)$. We will also use $\mathrm{L}[\cdot]$ to denote a general linear relation. For example, $P_{t}=\mathbf{L}\left[\underline{Z}_{t}\right]$ means that $P_{t}$ is a linear function of $\underline{Z}_{t}$. Since we often do not care about the actual functional form within the linear class, the same symbol is used for different functions. For example, no time subscript is attached, even though the actual function may depend on $t$. In addition, for variable $Z_{t}$ let $\Delta Z_{t}$ denote its first difference $Z_{t}-Z_{t-1}$. 


\subsection{Stock Market Equilibrium}

Given the well known properties of CARA preferences under normal distributions of payoffs and signals, we only consider the linear equilibria of the economy. ${ }^{8}$ In a linear equilibrium, the equilibrium stock price can be expressed as a linear function of the state variables of the economy. In other words, we have

$$
P_{t}=\mathrm{L}\left[\Phi_{t}\right]
$$

The general history dependence of the equilibrium under differential information leads to diffculties in solving the equilibrium since the dimensionality of the state variables increases over time without bound.

In the current setting, however, the general history dependence can be simplified by properly choosing the state space. The equilibrium can be fully characterized by a small number of state variables which only include first order expectations. This allows us to solve for the equilibrium prices and trading volume. Our main result is presented in the following theorem.

Theorem 1 In a linear equilibrium of the economy, (1) the price function has the following form:

$$
P_{t}=\left(1-p_{\Pi, t}\right) \hat{\Pi}_{t}^{c}+p_{\Pi, t} \Pi-p_{\theta, t} \Theta_{t}=\left(1-p_{\Pi, t}\right) \hat{\Pi}_{t}^{c}+p_{\Pi, t} \xi_{t}
$$

where $\xi_{t} \equiv \Pi-\mu_{t} \theta_{t}$ and $\mu_{t} \equiv \frac{p_{\theta, t}}{p_{\Pi, t}}$

(2) The conditional expectations are determined by the following linear filtering equations:

$$
\left(\begin{array}{c}
\hat{\Pi}_{t}^{c} \\
\hat{\Theta}_{t}^{c}
\end{array}\right)=\left(\begin{array}{cc}
1 & 0 \\
0 & a_{\Theta}
\end{array}\right)\left(\begin{array}{c}
\hat{\Pi}_{t-1}^{c} \\
\hat{\Theta}_{t-1}^{c}
\end{array}\right)+K_{t}^{c}\left(\begin{array}{c}
\xi_{t}-E_{t-1}^{c}\left[\xi_{t}\right] \\
Y_{t}-E_{t-1}^{c}\left[Y_{t}\right]
\end{array}\right)
$$

and

$$
\left(\begin{array}{c}
\hat{\Pi}_{t}^{i} \\
\hat{\Theta}_{t}^{i}
\end{array}\right)=\left(\begin{array}{cc}
1 & 0 \\
0 & a_{\theta}
\end{array}\right)\left(\begin{array}{c}
\hat{\Pi}_{t-1}^{i} \\
\widehat{\Theta}_{t-1}^{i}
\end{array}\right)+K_{t}\left(\begin{array}{c}
\xi_{t}-\mathbf{E}_{t-1}^{i}\left[\xi_{t}\right] \\
Y_{t}-\mathbf{E}_{t-1}^{i}\left[Y_{t}\right] \\
S_{t}^{i}-\mathbf{E}_{t-1}^{i}\left[S_{t}^{i}\right]
\end{array}\right)
$$

(3) Investor i's optimal stock holding is given by

$$
X_{\imath}^{i}=\hat{\Theta}_{\imath}^{i}+h_{\imath}\left(\hat{\Pi}_{\imath}^{i}-\hat{\Pi}_{t}^{c}\right), \quad i \in \mathcal{I} .
$$

Here, $K_{t}^{c}, K_{t}$ and $h_{t}$ are, respectively, $(2 \times 2),(2 \times 3)$, and $(1 \times 1)$ constant matrices.

Before we provide a proof for Theorem 1, several comments on the nature of the equilibrium are in order. First, the equilibrium stock price depends only on three variables, $\Pi, \theta_{t}$ and $\hat{\Pi}_{t}^{c}$. The general history dependence of the equilibrium price as expressed in (7) is summarized by a 
single variable $\hat{\Pi}_{t}^{\text {e! }}$ This significantly simplifies the characterization of the equilibrium. Second, the system of these variables follows a Gaussian Markov process under public information or the information of individual investors. This allows simple solutions to investors' optimization problems. Third, (8) is an implicit equation for the equilibrium price, since $\widehat{\Pi}_{t}^{c}$ on the right hand side is an endogenous variable and depends on $P_{t}$ itself. This dependence is, however, linear, as given in (9). Solving $P_{t}$ explicitly from (8) is a trivial matter. With this clarification in mind, we will use (8) as our pricing equation in the remainder of the paper.

The equilibrium stock price depends on $\theta_{t}$ and the true value of $\Pi$. Although the random stock supply $\theta_{t}$ is independent from the stock's cash flows, it enters the price function because it affects the number of stock shares held by the investors, Hence, the total risk the economy has to bear [see, e.g., Grossman and Miller (1988) and Campbell and Kyle (1993)]. $I$ is not in any investor's information set. A priori, one would not expect it to appear directly in the price function. Instead, the price should depend on the average of individual expectations of $\Pi$, which in turn depends on the average of private signals. In the current set-up, however, there are an infinite number of investors, and their private signals have i.i.d. noise. By the Law of Large Numbers, the average of all private signals is $\Pi$. Therefore, the equilibrium price depends on the true value of $\Pi$ and is not affected by the noise in individual signals (see also Lemma 1 in the next section). ${ }^{9}$ Furthermore, in (8) the coefficients of $\Pi$ and $\hat{\Pi}_{\imath}^{c}$ add up to one. This is because a constant shift in $\Pi$ will shift both $\hat{\Pi}_{t}^{c}$ and $\hat{\Pi}_{t}^{i}$ as well as $P_{t}$ by the same constant. (Recall that the riskless rate is assumed to be zero.)

\subsection{Expectations, Stock Demand, and Market Clearing}

We now sketch a four-step proof for Theorem 1. First, we discuss some general properties of the linear equilibrium as defined by (7).Second, we derive the dynamics of investors' expectations in a linear equilibrium as defined by (7), and show how the infinite regress problem (i.e., forecasting the forecasts of others) is resolved in our setting. Next, we derive investors' optimal stock demands in a linear equilibrium. Finally, we show that by imposing the market-clearing condition, the equilibrium stock price is given in (8) and investors' optimal stock holdings in equilibrium are given by (11).

\section{A. Equilibrium Price Function}

Let us consider a general linear equilibrium as defined by (7). The vector of state variables $\Phi_{t}$ is defined as the whole history of exogenous shocks to the economy, including supply shocks, public announcements, and private signals. Under the symmetry among investors in the quality of their signals, the following lemma is immediate: 
Lemma 1 In a linear equilibrium, the price function can be expressed as follows:

$$
P_{t}=\mathbf{L}\left[\Pi, \underline{\Theta}_{t}, \underline{Y}_{t}\right] \text {. }
$$

Furthermore, we can rewrite $P_{t}$ as:

$$
P_{t}=\mathrm{L}\left[I, \Theta_{t}, Y_{t}, P_{t-1}, Y_{t-1}\right]
$$

Equation (12) says that the dependence of the price on investors' private signals results in the dependence on the true value of $I .^{10}$

Equation (13) expresses the current equilibrium price as a function of current exogenous variables and past public information including past prices and announcements. ${ }^{11}$ The history dependence of the equilibrium price reflects the fact that investors' expectations depend on their information, which includes past prices and announcements. This suggests the possibility of simplifying the price function by expressing it in terms of investors' conditional expectations instead of their information sets or the exogenous state variable.

Let us now consider the information content of equilibrium prices in a linear equilibrium. We can rewrite (13) as:

$$
P_{t}=a_{t}\left(I-\mu_{t} \Theta_{t}\right)+b_{t} Y_{t}+\mathbf{L}\left[\underline{P}_{t-1}, \underline{Y}_{t-1}\right]
$$

This implies that, given past prices and announcements as well as the current announcement, observing the current price is equivalent to observing $\xi_{t}=\Pi-\mu_{t} \Theta_{t}$, which is a linear combination of the two unknowns. Consequently, in a linear equilibrium the information set $\mathcal{F}_{t}^{c}=\left\{\mathcal{F}_{0}, \underline{P}_{t}, \underline{Y}_{t}\right\}$ is equivalent to the information set $\left\{\mathcal{F}_{0}, \underline{\xi}_{t}, Y_{t}\right\}$, i. e., $\left\{\mathcal{F}_{0}, \underline{P}_{t}, \underline{Y}_{t}\right\} \Leftrightarrow\left\{\mathcal{F}_{0}, \underline{\xi}_{t}, \underline{Y}_{t}\right\}$.

Our ultimate goal is to prove that in equilibrium, equation (14) reduces to (8). This is equivalent to showing that in (14), $b_{t} Y_{t}+\mathbf{L}\left[E_{t-1}, Y_{t-1}\right]=\mathrm{L}\left[\hat{\Pi}_{t}^{c}\right]$ and only three variables, $\Pi$, $\theta_{t}$ and $\widehat{\Pi}_{t}^{c}$, are sufficient to determine the equilibrium price. Put differently, the specific form of (8) imposes a particular structure on the general linear price function as expressed in (14), especially on its coefficients. Under this specific structure, the dependence of the current price on the history of the economy is compactly summarized by its dependence on $\hat{\Pi}_{t}^{c}$.

\section{B. Investors' Expectations}

In order to derive each investor's optimal stock demand, we have to solve the conditional expectations, given his information set. In a linear equilibrium (12), calculating the conditional expectations of the true state variables is a linear filtering problem, since all the signals are linear in the state variables, including endogenous signals such as prices. 
Utilizing the equivalence between the two information sets, $\left\{\mathcal{F}_{0}, \underline{P}_{t}, \underline{Y}_{t}\right\}$ and $\left\{\mathcal{F}_{0}, \underline{\xi}_{t}, \underline{Y}_{t}\right\}$, we can solve for conditional expectations of $\Pi$ and $\Theta_{t}$ based on, respectively, the common information $\mathcal{F}_{i}^{c}$ and each investor's information $\mathcal{F}_{i}, i \in \mathcal{I}$. The results on the first-order expectations, i.e., the expectations of the true values of $\Pi$ and $\theta_{t}$, are summarized in the following lemma, the proof of which can be found in Appendix A.

Lemma 2 Given the linear price function $(\gamma),\left(\hat{\Pi}_{\imath}^{c}, \hat{\Theta}_{l}^{c}\right)$ and $\left(\hat{\Pi}_{\imath}^{i}, \hat{\theta}_{l}^{i}\right)$ are determined by the following stochastic difference equations

$$
\begin{aligned}
& \left(\begin{array}{c}
\hat{\Pi}_{t}^{c} \\
\hat{\Theta}_{t}^{c}
\end{array}\right)=\left(\begin{array}{c}
\mathbf{E}_{t-1}^{c}[\Pi] \\
\mathbf{E}_{t-1}^{c}\left[\Theta_{t}\right]
\end{array}\right)+K_{t}^{c}\left(\begin{array}{c}
\xi_{t}-\mathbf{E}_{t-1}^{c}\left[\xi_{t}\right] \\
Y_{t}-\mathbf{E}_{t-1}^{c}\left[Y_{t}\right]
\end{array}\right) \\
& \left(\begin{array}{c}
\hat{\Pi}_{t}^{i} \\
\hat{\Theta}_{t}^{i}
\end{array}\right)=\left(\begin{array}{c}
\mathbf{E}_{t-1}^{i}[\Pi] \\
\mathbf{E}_{t-1}^{i}\left[\Theta_{t}\right]
\end{array}\right)+K_{t}\left(\begin{array}{c}
\xi_{t}-\mathbf{E}_{t-1}^{i}\left[\xi_{t}\right] \\
Y_{t}-E_{t-1}^{i}\left[Y_{t}\right] \\
S_{t}^{i}-\mathbf{E}_{t-1}^{i}\left[S_{t}^{i}\right]
\end{array}\right)
\end{aligned}
$$

and $\hat{\Pi}_{0}^{c}=\hat{\Pi}_{0}^{i}=\hat{\Theta}_{0}^{c}=\hat{\Theta}_{0}^{i}=0$. The conditional variances of $I$ and $\Theta_{t}$ are deterministic and given in Appendix $A$.

Remark: Note that $\mathrm{E}_{t-1}^{c}[\Pi]=\hat{\Pi}_{t-1}^{c}$ and $\mathrm{E}_{t-1}^{c}\left[\Theta_{t}\right]=a_{\theta} \hat{\Theta}_{t-1}^{c}$. Equation (15) can be expressed as a recursive equation of $\left\{\hat{\Pi}_{t}^{c}, \hat{\Theta}_{t}^{c}\right\}$ with the surprises in $\xi_{t}$ and $Y_{t}$ as innovations. Thus, under the information process generated by $\mathcal{F}_{t}^{c},\left\{\hat{\Pi}_{t}^{c}, \hat{\Theta}_{t}^{c}\right\}$ follows a Gaussian Markov process. Similarly, $\left\{\hat{\Pi}_{i}^{i}, \hat{\Theta}_{i}^{i}, \hat{\Pi}_{\imath}^{c}, \hat{\Theta}_{t}^{c}\right\}$ follows a Gaussian Markov process under the information process generated by $\mathcal{F}_{i} \cdot{ }^{12}$ Furthermore, note that $\xi_{t} \equiv \Pi-\mu_{t} \theta_{t} \subseteq \mathcal{F}_{t}^{c} \subseteq \mathcal{F}_{t}^{i}$. Thus, $\hat{\Pi}_{t}^{i}-\mu_{t} \hat{\Theta}_{t}^{i}=\hat{\Pi}_{t}^{c}-\mu_{t} \hat{\Theta}_{t}^{c}=$ $\hat{\Pi}_{t}^{c}-\mu_{t} \hat{\Theta}_{t}^{c}$.

In general, investor i's trading strategy depends not only on his first-order expectations but also on his higher-order expectations such as his suppositions about other investors' expectations. In the current model, however, the higher order expectations can be reduced to first-order expectations, allowing us to solve the equilibrium in closed-form. Let $\hat{\Pi}_{t} \equiv \int_{i} \hat{\Pi}_{t}^{i}$ be the market average of investors' expectations of $\Pi$, and $\hat{\bar{\Pi}}_{t}^{i} \equiv \mathbf{E}_{l}^{i}\left[\hat{\Pi}_{t}\right]$ be investor $i$ 's expectation of the market average expectation of $\Pi$. Then, $\hat{\hat{\Pi}}_{t} \equiv \int_{i} \hat{\hat{\Pi}}_{t}^{i}$ is the market average of investors' expectations of $\hat{\Pi}_{\imath}$. We have the following lemma, the proof of which can be found in Appendix B.

Lemma 3 Let $w_{t}^{-1} \equiv \frac{1}{\sigma_{S, 1}^{2}}+\cdots+\frac{1}{\sigma_{s, s}^{2}}$ and $\alpha_{t} \equiv \frac{w_{l}}{\sigma_{i}^{i}+w_{t}}$. Then,

$$
\hat{\Pi}_{t}^{i}=\alpha_{t} \hat{\Pi}_{t}^{c}+\left(1-\alpha_{t}\right) \hat{\Pi}_{t}^{p, i}, \quad \hat{\Pi}_{t}^{p, i}=w_{t} \sum_{\tau=1}^{t} \frac{S_{\tau}^{i}}{\sigma_{S, \tau}^{2}} .
$$

This implies that

$$
\hat{\Pi}_{t}=\alpha_{t} \hat{\Pi}_{t}^{c}+\left(1-\alpha_{t}\right) \Pi, \quad \hat{\hat{\Pi}}_{t}^{i}=\alpha_{t} \hat{\Pi}_{t}^{c}+\left(1-\alpha_{t}\right) \hat{\Pi}_{t}^{i}, \quad \hat{\hat{\Pi}}_{t}=\alpha_{t} \hat{\Pi}_{t}^{c}+\left(1-\alpha_{t}\right) \hat{\Pi}_{t} .
$$


The first part of the lemma states that investor i's expectation of $\Pi$, conditional on his information, is a weighted average of his first-order expectations, conditional only on the public information and only on his private signals, respectively. The second part of the lemma shows that the second-order expectations (of $\Pi$ ) are a weighted average of two first-order expectations: the expectation of $\Pi$ conditional only on public information and the expectation of $\Pi$ conditional on both the public and the private information. In other words, it is a linear function of the first-order expectations. It is then easy to see that investor i's higher-order expectations can also be expressed as a linear function of his first-order expectations. Consequently, the number of state variables necessary to characterize the equilibrium of this economy does not explode as it would in general.

Our model has a finite time horizon and a finite dimensionality of unknown variables. The history of the economy at any point in time is finite dimensional and the number of state variables needed to characterize the equilibrium is finite, although it increases over time without bound. This differs from the infinite horizon situation considered by Townsend (1983) where the infinite regress problem arises. However, the nature of the problem here is similar to the infinite regress problem. With heterogeneous information, the state of the economy generally depends on its whole history, since investors' expectations are based on the sample path of the economy. When we try to express the history dependence through the expectations of investors, a hierarchy of expectations is needed. In the current setting, we are able to show that a few layers of expectations are sufficient to characterize the equilibrium. In particular, two expectations (individual investors' conditional expectations and their expectations of the market average of individual expectations) span the space of expectations, even though the dimensionality of the economy increases with the lifetime of the economy. In the continuous-time limit of the current model (with finite horizon), the history of the economy becomes infinite dimensional. The situation is then very similar to that of Townsend (with discrete time and infinite horizon) and the infinite regress problem does arise in general. It can be shown that the form of our solution remains the same in the continuous-time limit and two first-order expectations span the space of expectations. In this case, our model provides an example where the infinite regress problem can be resolved by collapsing the whole hierarchy of expectations into a few low-order expectations. (The solution to the continuous-time limit of the current model is available from the authors on request.)

\section{Investors' Optimal Stock Demand}

Let $Q_{t+1} \equiv P_{t+1}-P_{t}$ be the excess return on one share of stock. An investor's optimal stock 
demand is given by the solution to the following optimization problem:

$$
\begin{gathered}
\max _{X_{t}^{i}} \mathbf{E}\left[-e^{-\lambda W_{T}^{i}} \mid \mathcal{F}_{t}^{i}\right] \\
W_{t+1}^{i}=W_{t}^{i}+X_{t}^{i} Q_{t+1} .
\end{gathered}
$$

We have the following lemma, the proof of which can be found in Appendix C.

Lemma 4 Let $\Psi_{t}$ be a Gaussian process, $P_{t}=L\left(\Psi_{t}\right)$ and $\Psi_{t}^{i}=E_{t}^{i}\left[\Psi_{t}\right]$. Suppose that $Q_{t}$ and $\Psi_{t}^{i}$, both measurable with respect to $\mathcal{F}_{i}$, are Gaussian processes under information $\left\{\mathcal{F}_{i}: 1 \leq t \leq T\right\}$ and $Q_{t+1}=A_{Q, t+1} \Psi_{t}^{i}+B_{Q, t+1} \epsilon_{t+1}^{i}$ $\Psi_{t+1}^{i}=A_{\Psi, t+1} \Psi_{t}^{i}+B_{\Psi, t+1} \epsilon_{t+1}^{i}$ where $A_{Q, t+1}, A_{\Psi, t+1}, B_{Q, t+1}, B_{\Psi, t+1}$ are constant matrices of proper order and $\epsilon_{t+1}^{i}$ is normal conditional on $\mathcal{F}_{\mathfrak{i}}$. Then, investor $i$ 's optimal stock demand has the linear form:

$$
X_{t}^{i}=\frac{1}{\lambda} F_{t} \Psi_{t}^{i}
$$

where $F_{\mathrm{t}}$ is a constant matrix of proper order.

Remark The lemma can be applied to the special cases when $\Psi_{t}=\left(1, \Pi, \underline{\Theta}_{t}, \underline{Y}_{t}\right)^{\top}, \Psi_{t}=$ $\left(1, \Pi, \Theta_{t}, Y_{t}, P_{t-1}, \underline{Y}_{t-1}\right)^{\top}$, and $\Psi_{t}=\left(1, \Pi, \Theta_{t}, \hat{\Pi}_{t}^{c}\right)^{\top}$, respectively.

Equations (4)-(4) specify that investor $i$ 's investment opportunities (i.e., expected future returns) are fully characterized by $\Psi_{i}^{i}$, which follows a Gaussian process. Equation (20) states that investor $i$ 's optimal stock demand is a linear function of $\Psi_{i}^{i}$.

\section{Proof of Theorem 1}

Now, we complete the proof of Theorem 1. We first show that market-clearing of the stock requires the equilibrium price to have the form (8). For simplicity, we present the proof in the case without public announcements. The extension to the case with public announcements is straightforward. We prove by induction.

It is easy to show that for $T-1,(8)$ is correct. Note that at $T-1$, there is one period to go and the economy is equivalent to a static one. Then,

$$
P_{T-1}=\hat{\Pi}_{T-1}-p_{\Theta, T-1} \Theta_{T-1}=\left(1-\alpha_{T-1}\right) \Pi+\alpha_{T-1} \hat{\Pi}_{T-1}^{c}-p_{\Theta, T-1} \Theta_{T-1} .
$$

Now suppose that (8) is true for $t=s+1, \cdots, T-1$. We want to show that it is also true for $t=s$. If the equilibrium price can be written in the form of (8) for $t=s+1, \cdots, T-1$, the dimension of the state variables required to characterize the excess return process [for $t>s$ ] will be greatly reduced. In particular, $\Psi_{t}=\left(1, \Pi, \Theta_{t}, \hat{\Pi}_{t}^{c}\right)^{\top}$ fully specifies future returns on the 
stock, and $\Psi_{i}^{i}=\left(1, \hat{\Pi}_{t}^{i}, \hat{\Theta}_{i}^{i}, \hat{\Pi}_{t}^{c}\right)^{\top}$ determines investor $i$ 's expectation of future returns given his information. Since $\Psi_{t}^{i}$ follows a Gaussian Markov process, we can substitute it in Lemma 4 for $t=s+1, \cdots, T-1$ to calculate investors' expected returns and stock demand. Furthermore, given that (8) holds for $t>s$, we need only $\hat{\Pi}_{t}^{i}, \hat{\Theta}_{t}^{i}, \hat{\Pi}_{t}^{c}$ and one additional variable, the current price $P_{s}$, to specify investors' expectations at $t=s$. Thus, we can let $\Psi_{s}=\left(1, \Pi_{s}, \Theta_{s}, \hat{\Pi}_{s}^{c}, P_{s}\right)^{\top}$. Substituting all these into Lemma 4, we have the following market-clearing condition for $t=s$ :

$$
\int_{i} X_{s}^{i}=\frac{1}{\lambda} F \cdot \int_{i} \Psi_{s}^{i}=\theta_{.}
$$

Solving for $P_{a}$, we have

$$
P_{s}=\mathrm{L}\left[\hat{\Pi}_{s}, \hat{\Pi}_{s}^{c}, \hat{\Theta}_{s}, \Theta_{s}\right]
$$

Since $\xi_{t} \in \mathcal{F}_{t}^{i}(t=1, \cdots, T-1)$, we have $\hat{\Pi}_{t}-\mu_{t} \hat{\Theta}_{t}=\xi_{t}=\Pi_{t}-\mu_{t} \theta_{t}$ or $\hat{\theta}_{t}=\theta_{t}+\left(\hat{\Pi}_{t}-\Pi\right) / \mu_{t}$. Furthermore, from Lemma 3 , we have $\hat{\Pi}_{t}=\alpha_{t} \hat{\Pi}_{t}^{c}+\left(1-\alpha_{t}\right) \Pi$. Thus, we can express $\hat{\Theta}_{s}$ and $\hat{\Pi}_{s}$ in terms of $\Pi, \Theta_{e}$ and $\hat{\Pi}_{s}^{c}$ and write

$$
P_{s}=\mathrm{L}\left[\Pi, \Theta_{s}, \hat{\Pi}_{s}^{c}\right]
$$

Hence, (8) is also true for $t=s$. This completes our proof of (8). The proof of (9)-(10) follows immediately from Lemma 2.

In order to prove (11), we apply Lemma 4 given (8). From (8) and (9-10), we can write the excess share returns as follows:

$$
Q_{t+1}=\left[\left(1-p_{\Pi, t+1}\right) \hat{\Pi}_{t+1}^{c}-\left(1-p_{\Pi, t}\right) \hat{\Pi}_{t}^{c}\right]+\left(p_{\Pi, \ell+1}-p_{\Pi, \ell}\right) \Pi-\left(p_{\theta, t+1} \Theta_{t+1}-p_{\Theta, t} \Theta_{t}\right)
$$

Investor $i$ 's expected excess share return can be expressed as

$$
\mathrm{E}\left[Q_{t+1} \mid \mathcal{F}_{t}^{i}\right]=e_{\theta, t+1} \hat{\Theta}_{t}^{i}+e_{\Pi, t+1}\left(\hat{\Pi}_{t}^{i}-\hat{\Pi}_{t}^{c}\right)
$$

where $e_{\Pi, t+1}$ and $e_{\theta, t+1}$ are constants (see Appendix D). Intuitively, $e_{\Pi, t}$ and $e_{\theta, t}$ should be positive. In the case of homogeneous information, it can be shown that $e_{\theta, t} \geq 0$ and the equality holds only when $a_{\theta}=1$. In the case of differential information, we are not able to sign $e_{\Pi, t}$ and $e_{\theta, t}$. For the numerical solutions obtained with a wide range of parameter values, $e_{n, t}$ and $e_{\theta, t}$ are both positive. In the following discussions, we will assume that this is the case.

Individual $i$ 's expected excess return has two components. The first component, $e_{\theta, t+1} \hat{\Theta}_{t}^{i}$, represents the excess return expected by the investors to accommodate the liquidity traders' demand. Note, however, that investors do not observe the actual supply of the stock. The premium they anticipate depends on their expectation of the stock supply instead of its true value. The second component, $e_{\Pi, t+1}\left(\hat{\Pi}_{t}^{i}-\hat{\Pi}_{t}^{c}\right)$, represents the expected gains based on the 
investor's private information. Investor $i$ has private information that is not fully reflected in the price. The difference between his expected value of the stock and what is reflected in the price gives the expected change in future prices as the true value is gradually revealed. To simplify notation, define $\Delta_{t}^{i} \equiv \hat{\Pi}_{t}^{i}-\widehat{\Pi}_{t}^{c}$. From (9) and (10), it is easy to show that $\left\{\hat{\Theta}_{t}^{i}, \Delta_{t}^{i}\right\}$ follows a Gaussian Markov process under the information $\left\{\mathcal{F}_{t}: 1 \leq t \leq T\right\}$. Thus, we can let $\Psi_{t}^{i}=\left(1, \hat{\Theta}_{t}^{i}, \Delta_{t}^{i}\right)^{\top}$ in Lemma 4 (see Appendix $C$ for more details).

Given the simple process of excess share returns, we can obtain investors' optimal stock demands by applying Lemma 4:

$$
X_{t}^{i}=d_{\theta, t} \hat{\Theta}_{t}^{i}+d_{\Delta, t} \Delta_{t}^{i}
$$

The intuition behind the stock demand function is simple. Note that $\Theta_{t}^{i}$ and $\Delta_{t}^{i}$ are the two variables that determine linearly investor $i$ 's expected excess returns for reasons discussed above. As a result, investor $i$ 's demand must depend on these two variables.

In order to prove that $d_{\theta, t}=1$, consider the market-clearing condition:

$$
\int_{i} X_{t}^{i}=d_{\theta, t} \hat{\theta}_{t}+d_{\Delta, t}\left(\hat{\Pi}_{t}-\hat{\Pi}_{t}^{c}\right)=\theta_{t} \text {. }
$$

Since $\hat{\Pi}_{t}-\mu_{t} \hat{\theta}_{t}=\Pi-\mu_{t} \theta_{t}$ and $\hat{\Pi}_{t}=\alpha_{t} \hat{\Pi}_{t}^{c}+\left(1-\alpha_{t}\right) \Pi$, we have

$$
0=\left[-\frac{\alpha_{t}}{\mu_{t}} d_{\theta, t}+\left(1-\alpha_{t}\right) d_{\Delta, t}\right]\left(\Pi-\hat{\Pi}_{t}^{c}\right)+\left(d_{\theta, t}-1\right) \theta_{t} .
$$

This leads to two equations with two unknowns:

$$
\left(d_{\theta, t}-1\right)=0, \quad-\frac{\alpha_{t}}{\mu_{t}} d_{\theta, t}+\left(1-\alpha_{t}\right) d_{\Delta, t}=0
$$

for $t=1,2, \cdots, T-2, T-1$. Equation (11) then follows where $h_{t}=\alpha_{t} /\left[\mu_{t}\left(1-\alpha_{t}\right)\right]$. Thus, we have completed the proof of Theorem 1. We will not provide a formal proof for the existence of solution to (23), which is a set of algebraic equations. When $\sigma_{\delta}=0$, such a proof is possible (available from the authors on request). When $\sigma_{\delta} \neq 0$, an existence proof is difficult to construct. For the parameters chosen in the examples below, we are able to solve it numerically.

\subsection{Computation of the equilibrium}

The proof of Theorem 1 suggests that the equilibrium price functions can be solved by a recursive procedure. Indeed, starting with an initial guess of $o_{T-1}$, we can solve the equilibrium price function at $T-1, T-2, \cdots$, recursively.

Specifically, for given $o_{T-1}$, we first find the equilibrium price function at $T-1$ (i.e., $p_{\Pi, T-1}$ and $\left.p_{\theta, T-1}\right)$ as follows: The investor's optimal demand function and the equilibrium price function at $T-1$ can be derived explicitly. The excess return at $T$ is $Q_{T}=\Pi+\delta-P_{T-1}$. Thus,

$$
Q_{T}=\mathrm{E}_{T-1}^{i}\left[Q_{T}\right]+\left(\Pi-\hat{\Pi}_{T-1}^{i}\right)+\delta
$$


Since there is only one period remaining, investor $i$ 's demand function at $T-1$ has the linear form

$$
\begin{aligned}
X_{T-1}^{i} & =\frac{1}{\lambda} \frac{E_{T-1}^{i}\left[Q_{T}\right]}{V_{T-1}^{i}\left[Q_{T}\right]} \\
& =\frac{1}{\lambda V_{T-1}^{i}\left[Q_{T}\right]}\left[\left(1-p_{\Pi, T-1}\right) \Delta_{T-1}^{i}+p_{\theta, T-1} \hat{\theta}_{T-1}^{i}\right]
\end{aligned}
$$

Let $o_{T-1}=V_{T-1}^{i}[\Pi]$. This gives

$$
d_{\theta, T-1}=\frac{p_{\theta, T-1}}{\lambda\left(o_{T-1}+\sigma_{\delta}^{2}\right)}, \quad d_{\Delta . T-1}=\frac{1-p_{\Pi, T-1}}{\lambda\left(u_{T-1}+\sigma_{\delta}^{2}\right)} .
$$

We conclude that

$$
p_{\theta, T-1}=\lambda\left(\sigma_{T-1}+\sigma_{\delta}^{2}\right), \quad p_{\Pi, T-1}=1-\alpha_{T-1} .
$$

Given the equilibrium price functions for $t=T-1$, we can calculate all the parameters needed for finding the equilibrium price function at $T-2$. For example, from appendices $A, B$ and $\mathrm{D}$, we can calculate $f_{T-1}, K_{T-1}$ and $K_{T-1}^{c}$, as a function of $p_{\Pi, T-1}$ and $p_{\theta, T-1}$. We can also calculate $o_{T-2}$ and $o_{T-2}^{\mathrm{c}}$ as a function of $o_{T-1}, p_{\Pi, T-2}$ and $p_{\theta, T-2}$. A fixed point solution for $p_{\Pi, T-2}$ and $p_{\theta, T-2}$ is obtained using (23). The procedure can be repeated for $T-3, T-4$, ... (More details can be found in Appendix D.)

Assuming that the fixed point solutions for $p_{\Pi, t}$ and $p_{\theta, t}$ exist for all $t$, we should obtain by the recursive procedure a series of price functions from time $t=1$ to $t=T-1$ as a function of the initial guess $o_{T-1}$. This series of price functions form an equilibrium if $o_{0}^{c}$ (updated according to (A.2) in Appendix D) is the same as $\sigma_{n}^{2}$. Otherwise, a numerical search algorithm can be used to find the right initial guess of $o_{T-1}$.

We emphasize that there may be multiple equilibria in our equilibrium model. When $\sigma_{\delta}=0$, however, one can show that there is a unique linear equilibrium. We discuss this case further in Section 3. Grundy and McNichols (1989) have shown that when $a_{\theta}=1$ and investors receive their private information only in the first period, there could be two equilibria. ${ }^{13}$ One equilibrium has the unique feature that prices reveal no new information and there is no trading after the first period. However, this equilibrium is not generic in the sense that it exists only when $a_{\theta}=1$ and does not appear when $a_{\theta}<1$ [see footnote 18 in Grundy and McNichols (1989)]. Since we are restricted to cases where $a_{\theta}<1$ in our analysis, we will ignore the possibility of this equilibrium in our future analysis.

\section{Behavior of Prices and Volume}

We now use our model to analyze the behavior of equilibrium prices and volume. In particular, we examine the different patterns of trading volume that emerge under different specifications 
of information flows. We also consider how private information is gradually impounded into the equilibrium prices through trading.

\subsection{The Benchmark Case: Homogeneous Information}

Before we examine how private information affects equilibrium prices and volume, let us consider the special case when investors have homogeneous information. For simplicity, suppose that the true value of $\Pi$ is known to all investors from the beginning (i.e., $\sigma_{S, 1}=0$ ). The remaining risk in the stock's payoff is $\delta$.

In this case, $\hat{\Pi}_{t}^{c}=\hat{\Pi}_{t}^{i}=\Pi$, and the equilibrium price of the stock at date $t$ has the form $P_{t}=\Pi-p_{\theta, t} \theta_{t}$. Here, $\Pi$ represents the fundamental value of the stock and $-p_{\theta, t} \Theta_{t}$ represents the risk premium. The risk premium gives the discount on the price to compensate investors for bearing the risk in the future payoff of the stock, which is proportional to the total number of shares $\theta_{t}$ investors have to hold. ${ }^{14}$ The parameter $p_{\theta, t}$ reflects the liquidity of the market, since it determines the price sensitivity to the amount of noise trading. $p_{\theta, t}$ increases with $\sigma_{\delta}$, the risk in future payoffs, and $\lambda$, the investors' risk aversion parameter. Following Kyle (1985), we can interpret $\frac{1}{p_{\theta .1}}$ as a measure of market liquidity. It can be shown that under homogeneous information, $p_{\theta, t}$ increases when the terminal date approaches (i.e., as $t$ goes to $T$ ). In the limit that $a_{\theta} \rightarrow 1, p_{\theta, t}$ becomes a constant over time. The intuition behind this is that, given that shocks in stock supply exhibit mean-reversion, the current shock will eventually reverse itself. When there are many trading dates left, the investors can accommodate the current shock by taking additional positions now and unwinding them later when the shock reverses itself. They earn excess returns from the market-making activity without necessarily increasing their exposure to the risk associated with underlying stock value. When there are fewer trading dates left, however, it is more likely that the investors will not be able to unwind their positions before the final resolution of uncertainty. Thus, it is more risky to accommodate the supply shocks. Consequently, a higher premium is demanded by the investors, $p_{\theta, t}$ is larger, and the market is less liquid.

Since the value of $\Pi$ is public information, the equilibrium price fully reveals the supply shock $\theta_{t}$. Thus, $\hat{\theta}_{t}^{i}=\theta_{t}$ and investors' optimal stock holding in equilibrium is $X_{i}^{i}=\theta_{t}$. Each investor holds a fair share of the stock that is supplied to the market. This simply reflects the investors' market-making activity in accommodating supply shocks. The equilibrium volume of trade at date $t$ in this case is:

$$
\nu_{i}^{*}=\int_{i}\left|\theta_{t}-\theta_{t-1}\right|=\left|\Theta_{t}-\Theta_{t-1}\right|
$$


The expected volume at date $t$ is then

$$
\mathbf{E}\left[\mathcal{V}_{t}^{*}\right]=\sqrt{\frac{2}{\pi} \operatorname{Var}\left[\Delta \Theta_{t}\right]}
$$

where $\Delta \Theta_{t} \equiv \theta_{t}-\theta_{t-1} .{ }^{15}$ Clearly, under homogeneous information, the volume is completely determined by the exogenous supply shocks. This volume reflects the non-informational trading in the model.

\subsection{The General Case: Differential Information}

Let us now consider the general case in which investors have private information. In the rest of this section, we provide some general discussions on equilibrium prices, investors' trading strategies and equilibrium trading volume. More detailed results are discussed in Section 4.

\section{A. Equilibrium Prices}

We first consider the equilibrium price. From the results in the previous section, the equilibrium price of the stock at date $t$ is

$$
P_{t}=\left[p_{\Pi, t} \Pi+\left(1-p_{\Pi, t}\right) \hat{\Pi}_{t}^{c}\right]-p_{\theta, t} \Theta_{t}
$$

A few comments about the equilibrium price are in order. It has two components: The first component is associated with investors' expectations of the stock's future payoffs, and the second component is associated with the risk adjustment, as in the case of homogeneous information. The first component is not simply proportional to the average of investors' expectations about the stock's underlying value, which is $\left(1-\alpha_{t}\right) \Pi+\alpha_{t} \hat{\Pi}_{t}^{c}$. This differs from the result in static settings [e. g., Diamond and Verrecchia (1981), Hellwig (1981), and Admati (1985)]. ${ }^{16}$ In the multi-period setting, investors follow dynamic trading strategies. They speculate on the changes in future prices, while in the static setting they only speculate on the terminal value of the stock. Dynamic trading strategies generate equilibrium prices that are different from those generated by static strategies [see also Grundy and McNichols (1989), Brown and Jennings (1989) and Shalen (1993)]. Lastly, even though the equilibrium has the Markov nature, in that it only depends on the current state variables (including investors' expectations), the values of these variables depend on the history of the economy. In particular, the current price depends on past prices.

Similar to our discussion on homogeneous information, $p_{\theta, t}$ characterizes the liquidity of the market. Two factors affect $p_{\theta, t}$. One is the uncertainty of the true value of the stock. The other is the number of trading opportunities remaining before the terminal date, when uncertainty is fully resolved. The less uncertain the value of the stock, the lower the premium 
investors demand, and the smaller is $p_{\theta, t}$. On the other hand, the fewer the trading opportunities left, the less willing the investors are to take positions, and the higher is $p_{\theta, t}$. In the case of homogeneous and perfect information, the uncertainty about the stock's liquidation value (i.e., $\left.\sigma_{\delta}\right)$ stays constant throughout the trading periods. The liquidity is determined purely by the second effect as discussed above. In the case of differential information, information about $\Pi$ is revealed over time through equilibrium prices. The revelation of information tends to decrease $p_{\theta, t}$, while the reduction of remaining trading opportunities tends to increase it. The resulting pattern of $p_{\theta, \ell}$ over time depends on the trade-off between these two factors.

Since the number of investors is infinite and the noise in their signals is i.i.d., the union of all private signals actually reveals $I I$. However, the supply shocks introduce noise into the prices. Thus, equilibrium prices only partially reveal investors' private information. The informativeness of the current price depends on the noise generated by the current supply shock, which equals $\mu_{\imath}^{2} \sigma_{\theta}^{2}$. As investors continue to trade, the sequence of prices reveals more information. The following corollary of Theorem 1 shows how informative the sequence of prices is about the true value of the stock. The proof of this corollary is contained in Appendix A.

Corollary 1 Let $f_{t}=1-a_{\theta} \frac{\mu_{1}}{\mu_{t-1}}$. Then

$$
\frac{1}{o_{t}^{c}}=\frac{1}{o_{t-1}^{c}}+\frac{f_{t}^{2}}{\mu_{t}^{2} \sigma_{\theta}^{2}}+\frac{1}{\sigma_{Y, t}^{2}}, \quad \frac{1}{o_{t}}=\frac{1}{o_{t-1}}+\frac{f_{t}^{2}}{\mu_{t}^{2} \sigma_{\theta}^{2}}+\frac{1}{\sigma_{Y, t}^{2}}+\frac{1}{\sigma_{S, t}^{2}}
$$

where $o_{t}^{c}$ and $o_{t}$, the conditional variance of $\Pi$ based on $\mathcal{F}_{t}^{c}$ and $\mathcal{F}_{t}$ respectively, decrease monotonically over time.

Thus, the amount of private information revealed through the prices increases with the number of trading rounds.

\section{$\underline{B . \text { Trading Strategies }}$}

Now, let us consider investors' trading strategies. Given the equilibrium conditions, investor $i$ 's equilibrium stock holding is

$$
X_{t}^{i}=\hat{\theta}_{t}^{i}+h_{t} \Delta_{t}^{i}, \quad h_{t}=\frac{\alpha_{t}}{\mu_{t}\left(1-\alpha_{t}\right)}>0 .
$$

It has two components. The first component is proportional to i's estimate of the supply shock. This component reflects investor $i$ 's position in accommodating the supply shocks. The second component is proportional to the difference between his estimation of the stock's underlying value and the estimation based purely on public information. This component reflects his speculative position based on his private information. When $\Delta_{t}^{i}=\hat{\Pi}_{t}^{i}-\hat{\Pi}_{t}^{c}>0$, the underlying value 
reflected in the price is lower than what investor $i$ expects. Thus, he may take a long position in the stock to capture expected future gains. The coefficient of the second component, $h_{t}$, characterizes the intensity of the investor's speculative trading. ${ }^{17}$

It is important to point out that although $h_{t} \Delta_{t}^{i}, i \in \mathcal{I}$, gives an investor's speculative position, the trading activity generated by differential information is not the simple sum of each investor's speculative activities. The above decomposition of an investor's position into marketmaking and speculative components is based on his own information. Given that investors have heterogeneous information, what is viewed as a non-informational trade by one investor could be viewed as an informational trade by another. ${ }^{18}$

In order to analyze investors' total trading activities associated with differential information, we define $x_{t}^{i} \equiv X_{i}^{i}-\theta_{t}$ which is investor $i$ 's position net of the per capita supply shock. Note that $\Theta_{t}$ is the position every investor takes as their market-making activity under homogeneous information. Thus, $x_{t}^{i}$ gives the part of investor $i$ 's position that is purely generated by differential information. Formally, we can write $X_{t}^{i}=\theta_{t}+x_{t}^{i}$. $\theta_{t}$ gives the non-informational trading and $x_{\imath}^{i}$ gives the informational trading. ${ }^{19}$ From Theorem 1, equation (23) and Lemma 3 , we can show that

$$
x_{t}^{i}=\frac{1}{\mu_{t}}\left(\hat{\Pi}_{t}^{p, i}-\Pi\right) .
$$

Thus, $x_{t}^{i}$ depends only on investor $i$ 's private information. Given an investor's private information, $\frac{1}{\mu_{e}}$ measures the amplitude of his informational trading. As the state of the economy changes, investors trade to revise both their speculative and their market-making positions. The volume generated by investor $i$ 's informational trading, given by $\left|x_{t}^{i}-x_{t-1}^{i}\right|$, not only depends on the new private information he receives, but also on changes in $\frac{1}{\mu_{l}} \equiv p_{\Pi, t} / p_{\Theta, t}$, the amplitude of his informational trading.

\section{Equilibrium Volume of Trade}

Given investors' optimal stock holdings, the equilibrium volume of trade at date $t$ is ${ }^{20}$

$$
\mathcal{V}_{t} \equiv \frac{1}{2} \int_{i}\left|X_{t}^{i}-X_{t-1}^{i}\right|+\frac{1}{2}\left|\Theta_{t}-\theta_{t-1}\right| \quad t=1, \cdots, T-1
$$

Subtract the volume under homogeneous information $\mathcal{V}_{t}^{*}$ from the total volume, we have:

$$
V_{t} \equiv \mathcal{V}_{t}-\mathcal{V}_{t}^{*}
$$

We will use $V_{t}$ as the measure of volume for our analysis. It is clear that $V_{t}$ is the volume generated by heterogeneous information in the current model. We can interpret $V_{t}$ as the volume of informational trading. From the definition of $x_{t}^{i}$ we have

$$
V_{t}=\frac{1}{2} \int_{i}\left|\Delta \theta_{t}+\Delta x_{t}^{i}\right|-\frac{1}{2}\left|\Delta \theta_{t}\right|
$$


and from (27), $\Delta \Theta_{t}$ and $\Delta x_{t}^{i}$ are uncorrelated, so

$$
\bar{V}_{t} \equiv \mathbf{E}\left[V_{t}\right]=\frac{1}{\sqrt{2 \pi}}\left(\sqrt{\operatorname{Var}\left[\Delta \Theta_{t}\right]+\operatorname{Var}\left[\Delta x_{t}^{i}\right]}-\sqrt{\operatorname{Var}\left[\Delta \Theta_{t}\right]}\right)
$$

The expected volume of informational trading will be the focus of our analysis. We present in the next section numerical results which illustrate the behavior of volume. Note that $V_{t}=0$ when $\forall i, \Delta x_{t}^{i}=0$.

\subsection{The Special Case with $\sigma_{\delta}=0$}

In general, it is difficult to study the equilibrium prices and volume analytically. However, when $\sigma_{\delta}=0$, we can explicitly characterize the equilibrium, and the behavior of prices and volume is greatly simplified. This is the situation considered in many existing models, since it has a simple solution [e.g., Brown and Jennings (1989), and Kim and Verrecchia (1991a,b)].

When $\sigma_{6}=0$, there will be no residual risk in the underlying value of the stock. If the number of trading dates is large, the true value of the stock will be almost fully revealed by the last trading date $T-1$ (i.e., $o_{T-1}^{c} \rightarrow 0$ if $T \rightarrow \infty$ ). More importantly, with $\sigma_{\delta}=0$ we can find the equilibrium in closed-form. We present the results as a corollary of our main theorem in Section 2, the proof of which can be found in Appendix E.

Corollary 2 Suppose that $\sigma_{\delta}=0$. Then, (1)

$$
\mu_{t} \equiv \frac{p_{\theta, t}}{p_{\pi, t}}=\lambda w_{t}, \quad X_{t}^{i}=\hat{\theta}_{t}^{i}+\frac{1}{\lambda \theta_{t}^{c}} \Delta_{t}^{i}
$$

for $t=1, \cdots, T-1$. In particular, if $\sigma_{S, t}^{2}=\sigma_{S}^{2}$ (constant over time), then $\mu_{t}=\frac{\lambda \sigma_{S}^{2}}{t}$;

(2) The expected equilibrium volume of informational trading is

$$
\bar{V}_{t}=\frac{1}{\sqrt{2 \pi}}\left(\sqrt{\operatorname{Var}\left[\Delta \Theta_{t}\right]+\frac{1}{\lambda^{2} \sigma_{S, t}^{2}}}-\sqrt{\operatorname{Var}\left[\Delta \Theta_{t}\right]}\right)
$$

where $\operatorname{Var}\left[\Delta \theta_{t}\right]=\frac{2 \sigma_{\theta}^{2}}{1+a_{\theta}}$.

Note that from the price, investors can infer $\Pi-\mu_{t} \theta_{t}$ which serves as a signal for the unknown value of the stock, $\Pi$. According to this corollary, the noise-signal ratio $\mu_{t}$ decreases over time when there is new private information every period. Thus, the current price becomes more and more informative about the true value of the stock.

Corollary 2 implies that informational trading occurs only when investors receive new private information. $\nabla_{t}$ is non-zero only if $\sigma_{S, t}^{2}$ is finite. Suppose that at date $t$, there are no private signals received by the investors. Then, $\sigma_{S, t}^{2}=\infty$ and $\bar{V}_{t}=0$. One may want to compare this equilibrium with the no-trading equilibrium of Grundy and McNichols (1989). The difference 
here is that even though there is no trading (other than the non-informational part), prices still reveal new information (when $a_{\theta} \neq 1$ ). The situation here reduces to the Grundy-McNichols no-trade equilibrium only when $a_{\theta}=1$ and investors receive private information only in the first period.

The above result extends the result of Pfleiderer (1984) obtained in a static setting to a multi-period setting. Even though there are many rounds of trading and investors maximize lifetime utility in the current setting, the static nature of investors' trading strategies in this special case $\left[\sigma_{\delta}=0\right]$ gives rise to this result. ${ }^{21}$

\section{Volume, Price Volatility, and Information}

We now examine in more detail the behavior of trading volume and its relation to price volatility under different specificaticns of the information flow in the market. Since the case where $\sigma_{\delta}=0$ was solved in closed-form in the previous section, we focus mainly on the general case where $\sigma_{\delta} \neq 0$, i.e., the case when all the combined private information in the market does not fully reveal the true value of the stock. In this case, we do not have closed-form solutions to the coefficients of the price function, and we resort to numerical methods.

Although our general model allows investors to receive new private information every period, we focus on the simple case where investors receive their private information only at the first date, i.e., $\sigma_{S, 1}<\infty$ and $\sigma_{S, \ell}=\infty$ for $1<t \leq T-1$. In addition to private information, investors may receive public information (other than prices) at given dates. The analysis of this case provides the basic intuition about the behavior of trading volume and its relation to price changes under differential information. We present various patterns of volume and price volatility for this case. While specific patterns of volume and price volatility may vary with the parameter values chosen, the qualitative features of those patterns are robust.

When investors receive their private information at date $t=1$, they establish speculative positions. The volume generated by the private information at date $t=1$, however, depends on the investors' initial stock positions. Since our main concern is the volume in subsequent periods, we will exclude the volume at $t=1$ from our future discussions thus avoiding making specific assumptions about investors' initial positions.

Our main findings are as follows: Even when there is no new information, private or public, after the first date, trading persists throughout the whole trading horizon. Investors establish their speculative positions when they first receive their private information, and then gradually unwind their positions. The unwinding of their positions can generate a non-monotonic volume pattern over time, which peaks in the middle of the trading horizon. When there are public 
announcements, investors increase their positions right before the announcement to speculate on the outcome of the announcement, and close their positions after the announcement. This generates high volume around the announcement date. The volume pattern over time, the amount of volume generated, and the total amount of information revealed through trading all depend on the timing of the announcement. Market liquidity drops right before the announcement and bounces back afterward. We also find that new information, private or public, generates both high volume and large price changes, while existing private information can generate high volume with little price changes.

\subsection{Volume and Private Information}

Let us first examine the dynamic behavior of expected trading volume when there are no public announcements before the terminal date. In this case, all the trading volume is generated by the private information received at $t=1$.

When $\sigma_{\delta}=0$, the volume of informational trading will be zero after the first date, as pointed out in the previous section. In particular, investors establish speculative positions at the first date based on their private information. At the following dates, they trade only to accommodate the supply shocks while maintaining their original speculative positions. ${ }^{22}$ This result might be surprising, since as time passes, more private information is revealed through investors' non-informational trading. This is illustrated in Corollary 1. The informativeness of the equilibrium price sequence increases over time even though there is no exogenous information coming to the market. This implies that the expected gains from speculation based on private information decrease over time. However, investors do not reduce their speculative positions. This is because as more information is impounded into the price, there is less risk associated with speculation and investors are willing to take larger positions. If we look at how $h_{t}$, as a measure of the intensity of investors' speculative trading, changes over time, we notice that $h_{t}$ increases monotonically over time when $\sigma_{\delta}=0$.

When $\sigma_{\delta} \neq 0$, volume does not drop to zero after the first period. As a matter of fact, volume can be non-monotonic over time, first increasing and then decreasing. In general, the expected trading volume at each date depends on all the private information received up to and including that date. Thus, the behavior of trading volume becomes much more complex than in the case where $\sigma_{\delta}=0$.

In the case where investors receive private information only on the first date, trading continues on the following dates. From (27), we have

$$
x_{t}^{i}=\frac{1}{\mu_{t}}\left(\hat{\Pi}_{t}^{p, i}-\Pi\right)=\frac{1}{\mu_{t}}\left(S_{1}^{i}-\Pi\right) .
$$


The volume of informational trade simply depends on $\operatorname{Var}\left[\Delta x_{t}^{i}\right]=\sigma_{S, 1}^{2}\left(\frac{1}{\mu_{t}}-\frac{1}{\mu_{t}-1}\right)^{2}$ which is completely determined by the dynamics of $\mu_{t}$ [see (30)]. In Figure 1, we show the pattern of expected trading volume for some parameter values. Here, we have chosen $T=50$ to be the total number of trading dates. The volume at the first date is omitted in the Figure for reasons mentioned earlier, and so is the volume at the terminal date, which is trivial. We see that volume exhibits a peak in the middle of the trading horizon. Also, note that volume jumps right before the terminal date. In general, details of the volume pattern depend on the parameter values. When $\sigma_{\delta}$ is very large, volume may decrease monotonically over time. When $\sigma_{\delta}$ is very small, volume may remain small until the last few trading dates and then increase. The pattern of volume over time also depends on the values of other parameters, $\lambda, a_{\theta}, \sigma_{\theta}$, etc., but the qualitative features as presented in Figure 1 are robust.

In order to understand the time pattern of trading volume, let us examine investors' trading strategies. Two factors are at play here. On the one hand, investors' information about the value of the stock becomes more accurate towards the terminal date; hence they trade more aggressively. Thus they maintain a large speculative position, even though the difference among them diminishes as more information is impounded into the price. This phenomenon is best seen in the case with $\sigma_{\delta}=0$ as discussed earlier, where $h_{t}$ increases monotonically over time. On the other hand, trading opportunities diminish closer to the terminal date. Investors become less willing to keep large positions, since it becomes more difficult to unload these positions. The residual risk $\delta$ plays a crucial role here. Since no investor has any information about $\delta$, an investor will bear this risk if he holds his position until the terminal date. In order to avoid this undesirable situation, it is optimal for investors to have relatively small speculative positions before the terminal date. In a dynamic setting with this expectation for his position at $T-1$, an investor will unwind his position before the terminal date, which generates trading along the way.

In Figure 2, we plot $h_{t}$ against time. We show (for some parameter values) that $h_{t}$ increases in the few periods after the first date, and then decreases, reflecting the trade-off between these two factors. When $\sigma_{\delta}$ is large (with respect to the remaining uncertainty in $\Pi$ at $T-1$, which can be measured by $o_{T-1}^{c}$, the effect of the residual risk dominates, and $h_{t}$ simply decreases monotonically over time. On the other hand, when $\sigma_{\delta}$ is small, $h_{t}$ may keep increasing until a few periods before the terminal date, and then decrease, reflecting investors' aggressive speculation during most of the trading horizon. $h_{t}$ always drops at $T-1$, right before the terminal date. This results in the high volume at date $T-1$. In the limit when $\sigma_{\delta}=0$, we reduce to the case where $h_{t}$ monotonically increases in time (even at $T-1$ ), $\overline{\left|x_{t}^{i}\right|}$ stays constant and there is no 
volume after $t=1$. For comparison, we have also plotted $h_{t}$ when $\sigma_{\delta}=0$ in Figure 2.

Although $h_{t}$ measures the intensity at which investors speculate on their private information, their actual positions also depend on their anticipated gains as well as their expectations about the supply shock. Figure 3 shows the investors' mean absolute position (net of the supply shock):

$$
\overline{\left|x_{t}\right|} \equiv \mathbf{E}\left[\left|x_{t}^{i}\right|\right]=\sqrt{\frac{2}{\pi} \operatorname{Var}\left[x_{i}^{i}\right]}
$$

where $\operatorname{Var}\left[x_{t}^{i}\right]=\frac{\sigma_{s, 1}^{2}}{\mu_{i}^{2}}$. For most parameter values, investors' mean absolute positions due to their informational trading are highest at the beginning of the trading horizon when they first receive their private information, and then decreașe gradually. For some parameter values, especially when $\sigma_{\delta}$ is small with respect to the remaining uncertainty in $\Pi$ at $T-1$ and investors' private signals have very low signal to noise ratio, both $h_{t}$ and $\overline{\left|x_{t}^{i}\right|}$ can increase over time until the last few periods. This situation arises when the first factor dominates.

It is clear that trading occurs when investors' positions change. The highest volume (after the initial date) occurs in the middle of the horizon, when investors greatly reduce their positions associated with informational trading. The position of the peak in volume depends on the residual risk $\sigma_{\delta}$. The peak occurs earlier as the residual risk increases. When $\sigma_{\delta}=0$, Figure 3 shows that $\overline{\left|x_{t}^{i}\right|}=0.25$ is constant over time, as pointed out earlier. ${ }^{23}$

\subsection{Volume and Public Information}

An abnormally high level of trading has been documented around public announcements such as earnings announcements, [see, e. g., Beaver (1968) and Bamber (1986)]. Several authors have considered the link between abnormal trading in response to public announcements and information heterogeneity among investors. ${ }^{24}$ For example, Kim and Verrecchia (1991a) have shown in a three-date setting, abnormal trading occurs only if there is some type of asymmetry among investors, either in their risk aversion or private information. In their model, public information does not generate trading volume when there is perfect symmetry among investors.

Our model generalizes the model of Kim and Verrecchia (1991a) to a general dynamic setting. The case with $\sigma_{\delta}=0$ is a direct extension of their model. With perfect symmetry among the investors in terms of their risk aversion and signal quality, public news does not generate any abnormal trading. As pointed out earlier, the volume of informational trading is completely determined by the new private information.

In the case where $\sigma_{\delta} \neq 0$, however, exogenous public information does generate trading. Figure 4 shows the pattern of volume when there is a public announcement. We have chosen $T=50$ as in the previous Figures, and the announcement date $t_{A}$ to be 8 or 30 . We want to 
compare this volume pattern with the volume pattern in absence of the public announcement, illustrated in Figure 1 (for the same parameter values). Without the announcement, the volume exhibits a peak at $t=25$ due to the endogenous informational trading among the investors. With the announcement, high volume is observed on the announcement date. For $t_{A}=8$, the announcement date comes before the peak of endogenous informational trading. The abnormal volume due to the announcement does not greatly affect the endogenous informational trading. Thus, a smooth peak occurs at $t=25$ due to endogenous informational trading and a sharp peak right on the announcement date, $t=8$. For $t_{A}=30$, the announcement date is close to the peak of endogenous informational trading. The announcement not only generates high volume on the announcement date, but also afects the pattern of endogenous trading. As a matter of fact, in this case, most of the trading is clustered around the announcement. Furthermore, the volume also depends on the timing of the announcement. For example, the volume on the announcement date is about 150 times larger when the announcement date $t_{A}=30$ than when $t_{A}=8$. Even if we add up the volume over the whole trading horizon, it is still much higher when $t_{A}=30$ than when $t_{A}=8$. Thus, the volume pattern heavily depends on the timing of the announcement.

The expected announcement can induce investors to time their trade around the announcement date. Let us consider the intensity of investors' speculative trading as measured by $h_{t}$. As figure 5 shows, $h_{t}$ jumps right before the announcement date and then declines. It is interesting to note that when the announcement is at $t_{A}=8, h_{t}$ jumps at $t=8$. Investors speculate a lot more aggressively after the announcement, since the risk of speculation is greatly reduced. When $t_{A}=30$, however, $h_{t}$ drops drastically after the announcement, since it is too close to the terminal date and investors do not want to carry a large speculative position. We can further examine the investors' mean absolute positions of informational trading, which are characterized by $\overline{\left|x_{t}\right|}$. Figure 6 shows that in both cases $\left(t_{A}=8\right)$ or 30 , investors take aggressive positions before the announcement date, and cut back their positions after the announcement. This pattern in their position taking clearly reflects the betting on the outcome of the announcement by the investors. When the announcement is early during the trading horizon $\left(t_{A}=8\right)$, investors carry large speculative positions already and the additional position taken before the announcement is small compared with the established positions. When the announcement is late $\left(t_{A}=30\right)$, investors have already reduced their speculative positions. The additional position taken before the announcement is very large compared with the established positions. ${ }^{25}$

It is interesting to note the difference in investors' behavior before the announcement date and before the terminal date. At both dates, part of the uncertainty about $\Pi$ is resolved. As 
we have noted, investors make aggressive bets before the announcement, but reduce speculative positions before the terminal date. The reason behind this difference is that at the terminal date, not only the true value of $\Pi$ will be revealed, but also the value of $\delta$. Investors have no information about $\delta$. If they want to bet on the outcome of the final revelation about $\Pi$, they will have to bear the risk of $\delta$. When $\sigma_{\delta}$ is large (with respect to the remaining uncertainty in $\Pi$ ), the risk dominates the potential gains from speculation. Thus, investors cut back their speculative positions just before the terminal date. For the announcement before the terminal date, however, there is no extra risk involved in taking speculative positions. The only uncertainty is about the value of $\Pi$, which is exactly what investors want to bet on, given their private information. Thus, they increase their speculative positions just before the announcement. In the limiting case where $\sigma_{\delta} \rightarrow 0$, we obtain the result that investors hold their positions to the terminal date.

We can also examine the change in liquidity around public announcements. Figure 7 shows that $p_{\theta, t}$ increases right before the announcement, indicating decreased liquidity. The liquidity decreases before the announcement due to the intensive informational trading, and increases after the announcement due to the reduction in uncertainty by the public revelation. The decrease in liquidity prior to the announcement will cause the price to be more sensitive to supply shocks, thus increase price volatility. In Figure 8, we also show the time path of $p_{n, \ell}$.

Another interesting point to notice is that the total amount of information revealed through the prices depends on the timing of the public announcement. Figure 9 plots $o_{i}^{c}$ over time when the announcement is at $t_{A}=8$ or 30 , respectively. Note that $o_{i}^{c}$ measures the remaining uncertainty in $\Pi$, given the history of prices and public announcements. Clearly, more private information is revealed through the prices when $t_{A}=30$ than when $t_{A}=8$. This is not surprising, given the investors' trading behavior in these two cases, as shown in Figure 5 . When $t_{A}=30$, investors bet more aggressively on the outcome of the announcement and more private information is revealed through the prices.

\subsection{Volume and Price Volatility}

Since volume is closely related to the flow of information to the economy, several authors have suggested that volume can be used to gauge the information flow to the economy [see, e.g., Clark (1973), and Lamoureux and Lastrapes (1990, 1992)]. It is argued that periods of high trading volume should be the periods with clustering in new information. These should also be the periods with persisting high volatility in prices. Thus, volume provides a measure of the economic time with respect to which the information flow is uncorrelated.

This argument can be justified when there is no private information. When there is private information in the market, the information flow can be exogenous or endogenous. Different 
information flows give rise to different behaviors of volume and price. We now use our model to examine the relation between volume and price volatility under different types of information flow.

In the current model, exogenous information includes public announcements and new private signals about the value of the stock; endogenous information is the stock price that reveals the existing private information. As discussed in the previous subsections, new public information mainly generates trading in the current period. New private information not only generates trading in the current period, but also generates trading in future periods. This implies that when there is private information, independent arrival of new information can generate serially correlated volume.

The price changes whenever there is new information. Two things contribute to such price changes: Changes in investors' expectations about the value of the stock, which changes $\Pi_{i}^{c}$ and changes in the uncertainty in the stock, which changes the price function, namely, the values of $p_{\Pi, t}$ and $p_{\theta, t}$. For example, $p_{\theta, t}$ increases right before the announcement and decreases after the announcement, as shown in Figure 7. Figure 10 shows the pattern of price volatility when there is a public announcement at $t_{A}=8$ or 30 , respectively. Price volatility jumps up at the announcement date. There is also abnormally high volume on the announcement date as shown in Figure 4. Similar results also hold in the case of new private information. Thus exogenous information generates both high volume and price volatility.

In the case of endogenous information, there can be trading in the absence of exogenous information. In this case, the high volume of trade may not be accompanied by large price changes. Consider the case when $t_{A}=8$. High volume occurs at $t=8$ due to the announcement, and at $t=25$ due to the existing private information (see Figure 4). However, no abnormal price volatility is observed on the dates of high volume (around $t=25$ ) that is generated by the existing private information. This contrasts with the volume at $t=8$, which is accompanied by high volatility in prices. The intuition behind this result is simple. The trading associated with existing information is mainly generated by the dynamic adjustments of investors' speculative positions. There are no abrupt changes in investors' perception of the stock's underlying value or the risk associated with trading. As a result, there are no abrupt price changes accompanying the volume.

It is also interesting to note that in some cases, high volatility occurs even before the actual announcement. For example, in the case when $t_{A}=30$ the volatility increases at $t=29$ and then further increases at $t=30$ when the announcement arrives. ${ }^{26}$ The increase in volatility at $t=29$ is caused by the decrease in liquidity before the announcement, i.e., an increase in $p_{\theta, t}$, 
as shown in Figure 7. Since the shocks in liquidity traders' demand are exogenously specified and independent of endogenous changes in liquidity, the observed price volatility increases when liquidity decreases. If one allows the supply shocks to be price sensitive, it is quite possible that the price volatility may not increase much until the date of the announcement.

Our examples show that when there is private information, trading is not only related to the new exogenous information, private or public, but also related to the existing private information. The trading generated by exogenous information is accompanied by high price volatility, while the trading generated by the existing private information is not.

In the more general case when there is new private information after the first period, the pattern of volume becomes more complex. Every piece of new information generates immediate trading as well as trading in future dates. The resulting volume and volatility patterns depend on the specific flow of information and the parameter values.

\section{Concluding Remarks}

In this paper, we have considered a multi-period model of stock trading in which investors have differential information about the true value of the stock. We show that the volume pattern over time is closely related to the flow and the nature of the information, e.g., exogenous versus endogenous, and private versus public. In our model, exogenous information includes new private signals and public announcements; endogenous information is simply the market-clearing prices that are public. Private information not only generates trading in the current period, but also leads to possible trading in future periods; public information mainly generates trading in the current period. Moreover, volume generated by exogenous information, private or public, is always accompanied by large price changes, while volume generated by existing information is not.

To facilitate our analysis, we have made various assumptions to make the model tractable. Some of these assumptions can be weakened. For example, it is possible to allow investors to have different degrees of risk aversion and different levels of precision for the signals they receive at any one time. (However, we still maintain that the Law of Large Numbers continues to apply to the partial sums of investors' private signals.) The assumption that there are a countably infinite number of investors is important. Also, for simplicity we have assumed that investors' signals are independent. We can extend our model to the case of a common noise in all investors' signals [similar to the assumptions in Grundy and McNichols (1989)]. Most of our results will remain the same. In fact, the common noise plays the same role as the residual risk $\delta$ in the stock's underlying value. 
Concerning the predictions on the behavior of volume, our results rely on the assumption that non-informational trading in the market is exogenously specified. If the non-informational trading is generated by changes in the demands of liquidity traders, it is more plausible to let the liquidity traders choose the optimal timing for and the amount of their trades [see, e.g., Admati and Pfleiderer (1988)]. Endogenous liquidity trading potentially alters the results we have in the current model. Moreover, given that the non-informational part of the trading is exogenous, we are able to decompose trading into informational and non-informational parts. When non-informational trading is endogenous, such a dichotomy becomes ambiguous, since informational trading will generally affect the non-informational trading and vice versa [see, e.g., Wang (1994)]. We leave the discussion of these issues for future research. 


\section{Appendix}

\section{A. Proof of Lemma 2}

To derive the filtering equations (9) and (10), we use the results in the following lemma, the proof of which can be found in Liptser and Shiryayev (1974).

Lemma A.1 Let

$$
x_{t}=A_{t} x_{t-1}+B_{t} \epsilon_{x, t}, \quad y_{t}=H_{t} x_{t}+\epsilon_{y, t}, \quad t=1,2, \cdots .
$$

$x_{t}$ is the $n$-vector of state variables at $t, y_{t}$ is the m-vector of observations at $t . A_{t}, B_{t}$ and $H_{t}$ are respectively $(n \times n),(n \times k),(m \times n)$ constant matrices. $\left\{\epsilon_{x, t}, t=1, \cdots\right\}$ and $\left\{\epsilon_{y, t}, t=1, \cdots\right\}$ are respectively a $k$-vector and an m-vector white Gaussian sequence. $\epsilon_{x, t} \sim \mathcal{N}\left(0, Q_{t}\right), \epsilon_{y, t} \sim$ $\mathcal{N}\left(0, R_{t}\right)$ and $x_{0} \sim \mathcal{N}\left(\bar{x}_{0}, \Sigma_{x, 0}\right), x_{0},\left\{\epsilon_{x, t}\right\}$ and $\left\{\epsilon_{y, t}\right\}$ are independent. Let

$$
\hat{x}_{t}=\mathbf{E}\left[x_{t} \mid y_{\tau}: 1 \leq \tau \leq t\right], \quad O_{t}=\mathbf{E}\left[\left(x_{t}-\hat{x}_{t}\right)\left(x_{t}-\hat{x}_{t}\right)^{\top} \mid y_{\tau}: 1 \leq \tau \leq t\right] .
$$

Then,

$$
\begin{aligned}
& \hat{x}_{t}=A_{t} \hat{x}_{t-1}+K_{t}\left(y_{t}-H_{t} A_{t} \hat{x}_{t-1}\right) \\
& O_{t}=\left(I_{n}-K_{t} H_{t}\right)\left(A_{t} O_{t-1} A_{t}^{\top}+B_{t} Q_{t} B_{t}^{\top}\right) \\
& K_{t}=\left(A_{t} O_{t-1} A_{t}^{\top}+B_{t} Q_{t} B_{t}^{\top}\right) H_{t}^{\top}\left[H_{t}\left(A_{t} O_{t-1} A_{t}^{\top}+B_{t} Q_{t} B_{t}^{\top}\right) H_{t}^{\top}+R_{t}\right]^{-1}
\end{aligned}
$$

where $I_{n}$ is the $(n \times n)$ identity matrix.

We can now solve for the common filters, $\hat{\Pi}_{t}^{c}$ and $\hat{\Theta}_{t}^{c}$, by applying this lemma. Make the following substitution: $x_{t}^{\top}=\left(\Pi, \Theta_{t}\right), y_{t}^{\top}=\left(\xi_{t}, Y_{t}\right), \epsilon_{x, t}=\epsilon_{\theta, t}, \epsilon_{y, t}^{\top}=\left(0, \epsilon_{Y, t}\right)$, and

$$
A_{t}=\left(\begin{array}{cc}
1 & 0 \\
0 & a_{\theta}
\end{array}\right), \quad B_{t}=\left(\begin{array}{l}
0 \\
1
\end{array}\right), \quad H_{t}=\left(\begin{array}{cc}
1 & -\mu_{t} \\
1 & 0
\end{array}\right), \quad Q_{t}=\sigma_{\Theta}^{2}, \quad R_{t}=\left(\begin{array}{cc}
0 & 0 \\
0 & \sigma_{Y, \ell}^{2}
\end{array}\right) .
$$

Since $p_{\Pi, t}\left(\Pi-\hat{\Pi}_{t}^{c}\right)=p_{\theta, t}\left(\Theta_{t}-\hat{\Theta}_{t}^{c}\right)$, we have

$$
\mathbf{E}\left[\left(\Theta_{t}-\hat{\Theta}_{t}^{c}\right)\left(\Theta_{t}-\hat{\Theta}_{t}^{c}\right) \mid \mathcal{F}_{t}^{c}\right]=\frac{1}{\mu_{t}^{2}} o_{t}^{c}, \quad \mathbf{E}\left[\left(\Pi-\hat{\Pi}_{t}^{c}\right)\left(\Theta_{t}-\hat{\Theta}_{t}^{c}\right) \mid \mathcal{F}_{t}^{c}\right]=\frac{1}{\mu_{t}} o_{t}^{c} .
$$

Also define $f_{t} \equiv 1-a_{\theta} \frac{\mu_{t}}{\mu_{t-1}}$ and

$$
\begin{aligned}
& D_{t}^{c} \equiv f_{t}^{2} \sigma_{Y, t}^{2} o_{t-1}^{c}+\mu_{t}^{2} \sigma_{\theta}^{2}\left(o_{t-1}^{c}+\sigma_{Y, t}^{2}\right) \\
& k_{\Pi, t}^{c, Y} \equiv \frac{1}{D_{t}^{c}} \mu_{t}^{2} \sigma_{\theta}^{2} o_{t-1}^{c}, \quad k_{\Pi, t}^{c, \xi} \equiv \frac{1}{D_{t}^{c}} f_{t} \sigma_{Y, t}^{2} o_{t-1}^{c} \\
& k_{\theta, t}^{c, Y} \equiv \frac{1}{D_{t}^{c}} \mu_{t} \sigma_{\theta}^{2} o_{t-1}^{c}, \quad k_{\theta, t}^{c, \xi} \equiv-\frac{1}{D_{t}^{c}}\left[\mu_{t} \sigma_{\theta}^{2}\left(o_{t-1}^{c}+\sigma_{Y, t}^{2}\right)-a_{\theta} \frac{f_{t}}{\mu_{t-1}} \sigma_{Y, t}^{2} o_{t-1}^{c}\right] .
\end{aligned}
$$


According to the above lemma, we obtain

$$
\left(\begin{array}{c}
\hat{\Pi}_{t}^{c} \\
\hat{\Theta}_{t}^{c}
\end{array}\right)=\left(\begin{array}{c}
\hat{\Pi}_{t-1}^{c} \\
a_{\theta} \hat{\Theta}_{t-1}^{c}
\end{array}\right)+\left(\begin{array}{cc}
k_{\Pi, t}^{c, \xi} & k_{\Pi, t}^{c, Y} \\
k_{\Theta, t}^{c, \xi} & k_{\Theta, t}^{c, Y}
\end{array}\right)\left(\begin{array}{c}
\xi_{t}-\left(\hat{\Pi}_{t-1}^{c}-a_{\theta} \mu_{t} \hat{\Theta}_{t-1}^{c}\right) \\
Y_{t}-\hat{\Pi}_{t-1}^{c}
\end{array}\right)
$$

and

$$
\frac{1}{o_{t}^{c}}=\frac{1}{o_{t-1}^{c}}+\frac{f_{t}^{2}}{\mu_{t}^{2} \sigma_{\theta}^{2}}+\frac{1}{\sigma_{Y, t}^{2}}
$$

At time 0 , we have $\frac{1}{o_{0}^{c}}=\frac{1}{\sigma_{n}^{2}}$.

Now, consider $\hat{\Pi}_{t}^{i}$ and $\hat{\theta}_{i}^{i}$. The derivation is similar to that of the common filters. In applying the above lemma, we make the following substitution: $x_{t}^{\top}=\left(\Pi, \theta_{t}\right), y_{t}^{i \top}=\left(\xi_{t}, Y_{t}, S_{t}^{i}\right)$, $\epsilon_{x, t}=\epsilon_{\theta, t}, \epsilon_{y, t}^{\top}=\left(0, \epsilon_{Y, t}, \epsilon_{S, t}^{i}\right)$, and

$$
A_{t}=\left(\begin{array}{cc}
1 & 0 \\
0 & a_{\Theta}
\end{array}\right), B_{t}=\left(\begin{array}{l}
0 \\
1
\end{array}\right), H_{t}=\left(\begin{array}{cc}
1 & -\mu_{t} \\
1 & 0 \\
1 & 0
\end{array}\right), R_{t}=\left(\begin{array}{ccc}
0 & 0 & 0 \\
0 & \sigma_{Y, t}^{2} & 0 \\
0 & 0 & \sigma_{S, t}^{2}
\end{array}\right) .
$$

We then have

$$
\mathbf{E}\left[\left(\theta_{t}-\hat{\theta}_{t}^{i}\right)^{2} \mid \mathcal{F}_{t}^{i}\right]=\frac{1}{\mu_{t}^{2}} o_{t}, \quad \mathbf{E}\left[\left(\Pi-\hat{\Pi}_{t}^{i}\right)\left(\theta_{t}-\hat{\Theta}_{t}^{i}\right) \mid \mathcal{F}_{t}^{i}\right]=\frac{1}{\mu_{t}} o_{t} .
$$

Also define $D_{t} \equiv f_{t}^{2} \sigma_{Y, t}^{2} \sigma_{S, t}^{2} o_{t-1}+\mu_{t}^{2} \sigma_{\Theta}^{2}\left[\left(\sigma_{Y, t}^{2}+\sigma_{S, t}^{2}\right) o_{t-1}+\sigma_{Y, t}^{2} \sigma_{S, t}^{2}\right]$ and

$$
\begin{aligned}
& k_{n, t}^{Y} \equiv \frac{1}{D_{t}} \mu_{t}^{2} \sigma_{\theta}^{2} \sigma_{S, t}^{2} o_{t-1}, \quad k_{\Pi, t}^{S} \equiv \frac{1}{D_{t}} \mu_{t}^{2} \sigma_{\theta}^{2} \sigma_{Y, t}^{2} o_{t-1}, \quad k_{\Pi, t}^{\xi} \equiv \frac{1}{D_{t}} f_{t} \sigma_{Y, t}^{2} \sigma_{S, t}^{2} o_{t-1}, \\
& k_{\theta, t}^{Y} \equiv \frac{1}{D_{t}} \mu_{t} \sigma_{\theta}^{2} \sigma_{S, t}^{2} o_{t-1}, \quad k_{\theta, t}^{S} \equiv \frac{1}{D_{t}} \mu_{t} \sigma_{\theta}^{2} \sigma_{Y, t}^{2} o_{t-1}, \\
& k_{\theta, t}^{\xi} \equiv-\frac{1}{D_{t}}\left\{\mu_{t} \sigma_{\theta}^{2}\left[\left(\sigma_{Y, t}^{2}+\sigma_{S, t}^{2}\right) o_{t-1}+\sigma_{Y, t}^{2} \sigma_{S, t}^{2}\right]-a_{\theta} \frac{f_{t}}{\mu_{t-1}} \sigma_{S, t}^{2} \sigma_{Y, t}^{2} o_{t-1}\right\} .
\end{aligned}
$$

We have

$$
\left(\begin{array}{c}
\hat{\Pi}_{i}^{i} \\
\hat{\Theta}_{t}^{i}
\end{array}\right)=\left(\begin{array}{c}
\hat{\Pi}_{t-1}^{i} \\
a_{\theta} \hat{\Theta}_{t-1}^{i}
\end{array}\right)+\left(\begin{array}{ccc}
k_{\Pi, t}^{\xi} & k_{\Pi, t}^{Y} & k_{\Pi, t}^{S} \\
k_{\theta, t}^{\xi} & k_{\theta, t}^{Y} & k_{\theta, t}^{S}
\end{array}\right)\left(\begin{array}{c}
\xi_{t}-\left(\hat{\Pi}_{t-1}^{i}-a_{\theta} \mu_{t} \hat{\Theta}_{t-1}^{i}\right) \\
Y_{t}-\hat{\Pi}_{t-1}^{i} \\
S_{t}-\hat{\Pi}_{t-1}^{i}
\end{array}\right)
$$

and

$$
\frac{1}{o_{t}}=\frac{1}{o_{t-1}}+\frac{f_{t}^{2}}{\mu_{t}^{2} \sigma_{\theta}^{2}}+\frac{1}{\sigma_{Y, t}^{2}}+\frac{1}{\sigma_{S, t}^{2}} .
$$




\section{B. Proof of Lemma 3}

Let $\underline{S}_{t}^{i}=\left(S_{1}^{i}, S_{2}^{i}, \cdots, S_{i}^{i}\right)$ and $\mathcal{F}_{0}^{c}=\mathcal{F}_{0}$ denote the prior. Then, $\mathcal{F}_{t}^{p, i}=\mathcal{F}_{i}^{S^{i}}$. Applying the results on conditional normal distributions [see, e.g., Liptser and Shiryayev (1974)], we have

$$
\mathbf{E}\left[\Pi \mid \mathcal{F}_{t}^{i}\right]=\hat{\Pi}_{t}^{c}+\Gamma_{t}\left(\underline{S}_{t}^{i}-\mathbf{E}\left[\underline{S}_{t}^{i} \mid \mathcal{F}_{t}^{c}\right]\right),
$$

where $\Gamma_{t}=\operatorname{Cov}\left[\Pi, S_{i}^{i} \mid \mathcal{F}_{t}^{c}\right]^{\top} \operatorname{Cov}\left[S_{t}^{i}, S_{t}^{i} \mid \mathcal{F}_{l}^{c}\right]^{-1}$. Note that

$$
\begin{aligned}
& \mathbf{E}\left[\underline{S}_{i}^{i} \mid \mathcal{F}_{t}^{c}\right]=\hat{\Pi}_{t}^{c} \underline{e}_{t} \\
& \operatorname{Cov}\left[\Pi, \underline{S}_{t}^{i} \mid \mathcal{F}_{t}^{c}\right]=\operatorname{Cov}\left[\Pi, \Pi \mid \mathcal{F}_{t}^{c}\right] \underline{e}_{t} \\
& \operatorname{Cov}\left[\underline{S}_{t}^{i}, \underline{S}_{t}^{i} \mid \mathcal{F}_{t}^{c}\right]=\operatorname{Cov}\left[\Pi, \Pi \mid \mathcal{F}_{t}^{c}\right] \underline{e}_{t} \underline{e}_{t}^{\top}+\operatorname{diag}\left(\sigma_{S, 1}^{2}, \cdots, \sigma_{S, 2}^{2}\right)
\end{aligned}
$$

where $\underline{e}_{t}$ is the $(t \times 1)$ column vector of 1 's and diag is the diagonal matrix. Thus,

$$
\Gamma_{t}=o_{t}^{c} \underline{e}_{t}^{\top}\left[o_{t}^{c} \underline{e}_{t} \underline{e}_{t}^{\top}+\operatorname{diag}\left(\sigma_{S, 1}^{2}, \cdots, \sigma_{S, t}^{2}\right)\right]^{-1} \text {. }
$$

It can be verified that $\Gamma_{t}=\frac{o_{t}^{c}}{o_{t}^{c}+w_{1}}\left(\frac{1}{\sigma_{s, 1}^{2}}, \cdots, \frac{1}{\sigma_{s, t}^{2}}\right)$. Now, it is easy to show that

$$
\mathbf{E}\left[\Pi \mid \mathcal{F}_{t}^{i}\right]=\alpha_{t} \hat{\Pi}_{t}^{c}+\left(1-\alpha_{t}\right) \hat{\Pi}_{t}^{p, i}, \quad \text { where } \quad \hat{\Pi}^{p, i}=w_{t} \sum_{\tau=1}^{t} \frac{S_{\tau}^{i}}{\sigma_{S, \tau}^{2}} .
$$

and $\epsilon_{t+1}^{i} \mid \mathcal{F}_{t} \sim \mathcal{N}\left(0, \Sigma_{t+1}\right)$

\section{Proof of Lemma 4}

Suppose that $\Psi_{l}$ is a Gaussian process and $P_{t}=\mathrm{L}\left(\Psi_{l}\right)$. Clearly $Q_{t} \equiv P_{t}-P_{t-1}$ and $\Psi_{t}^{i} \equiv \mathrm{E}\left[\Psi_{l} \mid \mathcal{F}_{l}^{i}\right]$ are adopted to $\mathcal{F}_{i}$. Suppose also that

$$
Q_{t+1}=A_{Q, t+1} \Psi_{t}^{i}+B_{Q, t+1} \epsilon_{t+1}^{i}, \quad \Psi_{t+1}^{i}=A_{\Psi, t+1} \Psi_{\imath}^{i}+B_{\Psi, t+1} \epsilon_{i+1}^{i}
$$

where $A_{Q, t+1}, A_{\Psi, t+1}, B_{Q, t+1}, B_{\Psi, t+1}$ are constant matrices of proper order and $\epsilon_{\imath+1}^{i} \mid \mathcal{F}_{\imath}^{i} \sim$ $\mathcal{N}\left(0, \Sigma_{t+1}\right)$. We want to prove that investor $i$ 's optimal holding has the linear form. Let us consider investor $i$ 's optimization problem:

$$
\operatorname{Max}_{X_{t}^{i}} \mathrm{E}\left[-e^{-\lambda W_{T}^{i}} \mid \mathcal{F}_{i}^{i}\right] \quad \text { s.t. } W_{i+1}^{i}=W_{i}^{i}+X_{i}^{i} Q_{t+1}
$$

[see Wang (1994)]. Let $J\left(W_{l}^{i} ; \Psi_{\ell}^{i} ; t\right)$ be the value functicn, the Bellman equation for the optimization problem reads:

$$
\begin{aligned}
0=\operatorname{Max}_{X_{t}^{i}} & \left\{\mathbf{E}\left[J\left(W_{t+1}^{i} ; \Psi_{t+1}^{i} ; t+1\right) \mid \mathcal{F}_{t}^{i}\right]-J\left(W_{t}^{i} ; \Psi_{t}^{i} ; t\right)\right\} \\
\text { s.t. } & W_{t+1}^{i}=W_{t}^{i}+X_{i}^{i} Q_{t+1} \\
& J\left(W_{T}^{i} ; \Psi_{T}^{i} ; T\right)=-e^{-\lambda W_{T}^{i}}
\end{aligned}
$$


We assume that the value function has the form: $J\left(W_{i}^{i} ; \Psi_{i}^{i} ; t\right)=-e^{-\lambda_{t} W_{i}-\frac{1}{2} \Psi_{i}^{\top} U_{t} \Psi_{i}}$. It is straightforward to show that

$$
\begin{aligned}
& \mathbf{E}\left[J\left(W_{t+1}^{i} ; \boldsymbol{\Psi}_{t+1}^{i} ; t+1\right) \mid \mathcal{F}_{t}^{i}\right]=-\rho_{t+1} e^{-\lambda_{t+1} W_{t}-\frac{1}{2} \Psi_{t}^{\top} A_{\psi, t+1}^{\top} U_{t+1} A_{\psi, t+1} \Psi_{i}^{i}} \times \\
& e^{-\lambda_{t+1} X_{t}^{i} A_{Q, t+1} \psi_{i}^{i}+\frac{1}{2}\left(\lambda_{t+1} B_{Q, 1+1}^{\top} X_{t}^{\top}+B_{\psi, t+1}^{\top} U_{t+1} A_{\psi, t+1} \psi_{t}^{\top}\right)^{\top} \Xi_{t+1}\left(\lambda_{t+1} B_{Q, t+1}^{\top} X_{i}^{i}+B_{\psi, t+1}^{\top} U_{t+1} A_{\psi, t+1} \psi_{t}^{!}\right)}
\end{aligned}
$$

where $\Xi_{t+1} \equiv\left(\Sigma_{t+1}^{-1}+B_{\Psi, t+1}^{\top} U_{t+1} B_{\Psi, t+1}\right)^{-1}$ and $\rho_{t+1}=\sqrt{\Xi_{t+1}|| \mid \Sigma_{t+1}}$. Define

$$
\begin{aligned}
F_{t} \equiv & {\left[B_{Q, t+1} \Xi_{t+1} B_{Q, t+1}^{\top}\right]^{-1}\left(A_{Q, t+1}-B_{Q, t+1} \Xi_{t+1} B_{\Psi, t+1}^{\top} U_{t+1} A_{\Psi, t+1}\right), } \\
M_{t} \equiv & F_{t}^{\top}\left(B_{Q, t+1} \Xi_{t+1} B_{Q, t+1}^{\top}\right) F_{t}-\left(B_{\Psi, t+1}^{\top} U_{t+1} A_{\Psi, t+1}\right)^{\top} \Xi_{t+1}\left(B_{\Psi, t+1}^{\top} U_{t+1} A_{\Psi, t+1}\right)+ \\
& A_{\Psi, t+1}^{\top} U_{t+1} A_{\Psi, t+1} .
\end{aligned}
$$

It is easy to derive the following first-order condition with respect to $X_{t}$ :

$$
X_{t}^{i}=\frac{1}{\lambda_{t+1}} F_{t} \Psi_{t}^{i}, \quad(1 \leq t<T) .
$$

The second-order condition for optimality is: $B_{Q, t+1} \Xi_{t+1} B_{Q, t+1}^{\top}>0$. Furthermore,

$$
\mathbf{E}\left[J\left(W_{t+1}^{i} ; \Psi_{t+1}^{i} ; t+1\right) \mid \mathcal{F}_{t}^{i}\right]=-\rho_{t+1} e^{-\lambda_{t+1} W_{t}^{i}-\frac{1}{2} \Psi_{t}^{i} M_{t} \psi_{t}}
$$

Substitute eq. (C.2-C.3) into the Bellman equation, we obtain the following for $t<T$ :

$$
\lambda_{t}=\lambda_{t+1} \text {, and } U_{t}=M_{t}+c_{t} I_{11}^{4,4}
$$

where $c_{t} \equiv-2 \ln \rho_{t+1}$ and $I_{1 i}^{4,4}$ is a $(4 \times 4)$ index matrix which has all the elements being zero except element $\{11\}$ being 1 . From the solution for $T-1, \cdots, t+1$, we can recursively solve for $\lambda_{t}$, and $U_{t}$ hence the value function and the optimal investment policy. Hence, we have completed the proof of Lemma 4.

In a general linear equilibrium, $P_{t}=\mathbf{L}\left(\Psi_{t}\right)$ by Lemma 1 where $\Psi_{t}=\left(1, \Pi, \Theta_{t}, Y_{t}\right)^{\top} \cdot \Psi_{t}^{i}=$ $\mathbf{E}_{t}^{i}\left[\Psi_{t}\right]=\left(1, \hat{\Pi}_{t}^{i}, \hat{\Theta}_{t}^{i}, \underline{Y}_{t}\right)$. Since $\mathbf{E}_{t}^{i}\left[\Theta_{t+1}\right]=a_{\Theta} \hat{\Theta}_{t}^{i}$ and $\mathbf{E}_{t}^{i}\left[Y_{t+1}\right]=\hat{\Pi}_{t}^{i}$, we have $\mathbf{E}_{t}^{i}\left[P_{t+1}\right]=\mathrm{L}\left(\Psi_{t}^{i}\right)$. Thus

$$
\mathbf{E}_{t}^{i}\left[Q_{t+1}\right]=\mathbf{E}_{t}^{i}\left[P_{t+1}-P_{t}\right]=A_{Q, t+1} \Psi_{t}^{i}
$$

where $\Psi_{t}^{i}=\left(1, \hat{\Pi}_{t}^{i}, \hat{\Theta}_{t}^{i}, \underline{Y}_{t}\right)^{\top}$ and $A_{Q, t+1}$ is a constant matrix with proper order. Note that $\Psi_{t+1}^{i}=\left(1, \hat{\Pi}_{t+1}^{i}, \hat{\theta}_{t+1}^{i}, \underline{Y}_{t+1},\right)^{\top}$ and

$$
\mathbf{E}_{t}^{i}\left[\Psi_{t+1}^{i}\right]=\left(1, \hat{\Pi}_{t}^{i}, \hat{\Theta}_{t}^{i}, a_{\Theta} \hat{\Theta}_{t}^{i}, \underline{Y}_{t}, \hat{\Pi}_{t}^{i}\right)^{\top},
$$

we can write

$$
\mathbf{L}_{t}^{i}\left[\Psi_{t+1}^{i}\right]=A_{\Psi, t+1} \Psi_{t}^{i}
$$


where $A_{\Psi_{, t+1}}$ is a constant matrix of proper order. Furthermore, note that $\xi_{t} \subseteq \mathcal{F}_{t}^{c} \subseteq \mathcal{F}_{t}$. Thus,

$$
\hat{\Pi}_{t}^{i}-\mu_{t} \hat{\Theta}_{t}^{i}=\hat{\Pi}_{t}^{c}-\mu_{t} \hat{\Theta}_{t}^{c}=\Pi-\mu_{t} \Theta_{t} .
$$

Then from Lemma 2, it easy to show that $Y_{t+1}-E_{t}^{i}\left[Y_{\ell+1}\right], \hat{\Pi}_{t+1}^{i}-E_{t}^{i}\left[\hat{\Pi}_{t+1}^{i}\right], \hat{\Theta}_{t+1}^{i}-E_{t}^{i}\left[\hat{\Pi}_{t+1}^{i}\right]$ and $P_{t+1}-\mathbf{E}_{t}^{i}\left[P_{t+1}\right]$ are all linear in $\epsilon_{t+1}^{i}=\left(\Pi-\Pi_{t}^{i}, \epsilon_{\theta, t+1}, \epsilon_{Y, t+1}, \epsilon_{S, t+1}^{i}\right)^{\top}$. Under the normality assumption about all the underlying shocks, we have $\epsilon_{t+1}^{i} \mid \mathcal{F}_{t}^{i} \sim \mathcal{N}\left(0, \Sigma_{t+1}\right)$ where $\Sigma_{t+1}=$ $\operatorname{diag}\left(o_{t}, \sigma_{\Theta}^{2}, \sigma_{Y, t+1}^{2}, \sigma_{S, t+1}^{2}\right)$. Thus, the excess return process can be expressed in the form of (C.1). We can then apply the above result and conclude that $X_{i}^{i}$ is linear in $\Psi_{t}^{i}$.

In the case when $P_{t}=\mathrm{L}\left(\Psi_{t}\right)$ and $\Psi_{t}=\left(1, \Pi, \theta_{t}, \hat{\Pi}_{t}^{c}\right)$, the problem is even simpler since now $\Psi_{t}$ is (Gaussian) Markov. It is easy to verify that we have (C.1) in this case and the above result also applies.

\section{Calculating the Equilibrium}

We now use the results in Theorem 1 and Lemmas 1-4 to solve the coefficients in the equilibrium price function (8). Let us first derive the excess return process. From Lemma 2, we have $\hat{\Pi}_{t+1}^{c}=\widehat{\Pi}_{t}^{c}+k_{\Pi, t+1}^{c, Y}\left(Y_{t+1}-\mathbf{E}\left[Y_{t+1} \mid \mathcal{F}_{t}^{c}\right]\right)+k_{\Pi, t+1}^{c, \xi}\left\{\xi_{t+1}-\mathbf{E}\left[\xi_{t+1} \mid \mathcal{F}_{t}^{c}\right]\right\}$

$\mathrm{E}\left[\hat{\Pi}_{t+1}^{c} \mid \mathcal{F}_{t}^{i}\right]=\hat{\Pi}_{t}^{c}+\left(k_{n, t+1}^{c, Y}+f_{t+1} k_{\Pi, t+1}^{c, \xi}\right)\left(\hat{\Pi}_{t}^{i}-\hat{\Pi}_{t}^{c}\right) \cdot$ Asshownin AppendixC $\hat{\Theta}_{t}^{i}-\hat{\Theta}_{t}^{c}=\left(\hat{\Pi}_{t}^{i}-\right.$ $\left.\hat{\Pi}_{t}^{c}\right) / \mu_{t}$. Defining $b_{t+1}^{Y} \equiv\left(1-p_{\Pi, t+1}\right) k_{\Pi, t+1}^{c, Y}$ and $b_{t+1}^{\xi} \equiv\left(1-p_{\Pi, t+1}\right) f_{t+1} k_{\Pi, t+1}^{c, \xi}$ where $f_{t+1}, k_{\Pi, t+1}^{c, Y}$ and $k_{n, t+1}^{c, \xi}$ are defined in Appendix $A$, we can then express investor $i$ 's expectation of the excess share return as:

$$
\mathrm{E}\left[Q_{t+1} \mid \mathcal{F}_{t}^{i}\right]=e_{\Pi, t+1}\left(\hat{\Pi}_{t}^{i}-\hat{\Pi}_{t}^{c}\right)+e_{\theta, t+1} \hat{\Theta}_{t}^{i}
$$

where $e_{\Pi, t+1} \equiv\left(p_{\Pi, t+1}-p_{\Pi, t}\right)+b_{t+1}^{Y}+b_{t+1}^{\xi}, e_{\theta, t+1} \equiv-\left(a_{\theta} p_{\Theta, t+1}-p_{\theta, t}\right)$. Define $\Delta_{t}^{i} \equiv \hat{\Pi}_{t}^{i}-\hat{\Pi}_{t}^{c}$. $\Delta_{t}^{i}$ is the difference between investor-i's estimate of the stock value and the estimate based solely on market information. It is easy to show that $E\left[\Delta_{t+1}^{i} \mid \mathcal{F}_{t}^{i}\right]=\left(1-k_{\Pi, t+1}^{c, Y}-f_{t+1} k_{\Pi, t+1}^{c, \xi}\right) \Delta_{t}^{i}$.

Thus, the expected excess stock return for investor $i$ is driven by two state variables $\hat{\Theta}_{i}^{i}$ and $\Delta_{i}^{i}$. Furthermore, $\left(\hat{\Theta}_{\mathfrak{i}}^{i}, \Delta_{\mathfrak{i}}^{i}\right)$ follows a linear Markov process with respect to $\mathcal{F}_{\mathfrak{t}}$. We can then simply let $\Psi_{t}^{i}=\left(1, \hat{\theta}_{t}^{i}, \Delta_{i}^{i}\right)$ in applying Lemma 4 to solve the equilibrium. In particular, define $\delta_{t+1}^{Y} \equiv k_{\Pi, t+1}^{Y}-k_{\Pi, t+1}^{c, Y}, \delta_{t+1}^{\xi} \equiv k_{\Pi, t+1}^{\xi}-k_{\Pi, t+1}^{c, k}$. We have

$$
\begin{aligned}
A_{\Psi, t+1} & =\left(\begin{array}{ccc}
1 & 0 & 0 \\
0 & 1-k_{n, t+1}^{c, Y}-f_{t+1} k_{\Pi, t+1}^{c, \xi} & 0 \\
0 & 0 & a_{\theta}
\end{array}\right) \\
B_{\Psi, t+1} & =\left(\begin{array}{cccc}
0 & 0 & 0 & 0 \\
\delta_{t+1}^{Y}+k_{\Pi, t+1}^{S}+f_{t+1} \delta_{t+1}^{\xi} & -\delta_{t+1}^{\xi} \mu_{t+1} & \delta_{t+1}^{Y} & k_{\Pi, t+1}^{S} \\
k_{\theta, t+1}^{Y}+k_{\theta, t+1}^{S}+f_{t+1} k_{\theta, t+1}^{\xi} & -k_{\theta, t+1}^{\xi} \mu_{t+1} & k_{\theta, t+1}^{Y} & k_{\theta, t+1}^{S}
\end{array}\right),
\end{aligned}
$$


$A_{Q, t+1}=\left(0, e_{\Pi, t+1}, e_{\theta, t+1}\right)$ and $B_{Q, t+1}=\left(f_{t+1} p_{\Pi, t+1}+b_{t+1}^{Y}+b_{t+1}^{\xi},-\left[p_{\theta, t+1}+b_{t+1}^{\xi} \frac{\mu_{t+1}}{f_{t+1}}\right], b_{t+1}^{Y}, 0\right)$. Following Lemma 4 , we obtain the excess return process and investors' optimal stock demand. The market-clearing conditions then lead to algebraic equations (23) about the undetermined coefficients in the price function, $p_{n, t}$ and $p_{\theta, t}$, which can then be solved.

\section{E. Proof of Corollary 2}

We will only prove $\mu_{t}=\lambda w_{t}$, since the rest of the corollary is straightforward. For ease of exposition, we present our proof only for the case where $T=3, a_{\Theta}=0, \sigma_{Y}=\infty$ and $\sigma_{S, t}=\sigma_{S}$. It then follows that $f_{t} \equiv 1$. To prove the proposition for $t=2$, we recall from (24) that $p_{\theta, 2}=\lambda o_{2}$ and $p_{\pi, 2}=1-\alpha_{2}=\frac{o_{2}^{c}}{o_{2}^{c}+w_{2}}$. Since $o_{2}=\left(\frac{1}{o_{2}^{c}}+\frac{1}{w_{2}}\right)^{-1}=\frac{o_{2}^{c} w_{2}}{o_{2}^{c}+w_{2}}$, we conclude that $\mu_{2}=\lambda w_{2}$. In the rest of the proof, we will repeatedly use Corollary 1 as well as the following relationship

$$
\frac{1}{o_{t}}=\frac{1}{o_{t}^{c}}+w_{t}^{-1}
$$

to simplify terms. This relationship can be derived as follows,

$$
\begin{aligned}
o_{t} \equiv \mathbf{E}\left[\left(\Pi-\hat{\Pi}_{t}^{i}\right)^{2} \mid \mathcal{F}_{i}^{i}\right] & =\mathbf{E}\left[\left\{\alpha_{t}\left(\Pi-\hat{\Pi}_{t}^{c}\right)+\left(1-\alpha_{t}\right)\left(\Pi-\hat{\Pi}_{t}^{p, i}\right)\right\}^{2} \mid \mathcal{F}_{t}^{i}\right] \\
& =\alpha_{t}^{2} \mathbf{E}\left[\left(\Pi-\hat{\Pi}_{t}^{c}\right)^{2} \mid \mathcal{F}_{t}\right]+\left(1-\alpha_{t}\right)^{2} \mathbf{E}\left[\left(\Pi-\hat{\Pi}_{t}^{p, i}\right)^{2} \mid \mathcal{F}_{t}^{i}\right] \\
& =\alpha_{t}^{2} o_{t}^{c}+\left(1-\alpha_{t}\right)^{2} w_{t}
\end{aligned}
$$

Substituting (E.2) in the definition of $\alpha_{t}$ yields the claim.

We now prove the proposition for $t=1$. Simple (but tedious) calculations show that

$$
A_{\Psi, 2}=\left(\begin{array}{ccc}
1 & 0 & 0 \\
0 & 1-\frac{o_{2}^{c}}{\mu_{2}^{2} \sigma_{\theta}^{2}} & 0 \\
0 & 0 & 0
\end{array}\right), B_{\Psi, 2}=\left(\begin{array}{ccc}
0 & 0 & 0 \\
\frac{o_{2}}{\sigma_{S}^{2}}+\frac{1}{\mu_{2}^{2} \sigma_{\theta}^{2}}\left(o_{2}-o_{2}^{c}\right) & -\frac{1}{\mu_{2} \sigma_{\theta}^{2}}\left(o_{2}-o_{2}^{c}\right) & \frac{o_{2}}{\sigma_{S}^{2}} \\
-\frac{o_{2}}{\mu_{2} o_{1}} & o_{2}\left(\frac{1}{\sigma_{S}^{2}}+\frac{1}{o_{1}}\right) & \frac{o_{2}}{\mu_{2} \sigma_{S}^{2}}
\end{array}\right)
$$

Since $X_{2}^{i}=\frac{1}{\lambda}\left(0, \frac{1}{o_{2}^{c}}, \lambda\right) \underline{\Psi}_{2}^{i}$, we get

$$
\begin{aligned}
& U_{2}=o_{2}\left(\begin{array}{c}
0 \\
\frac{1}{\sigma_{2}^{c}} \\
\lambda
\end{array}\right)\left(0, \frac{1}{o_{2}^{c}}, \lambda\right), B_{\Psi, 2}^{\top} U_{2} B_{\Psi, 2}=o_{2}\left(\begin{array}{c}
-\frac{1}{\sigma_{S}^{2}} \\
\lambda \\
\frac{1}{\sigma_{S}^{2}}
\end{array}\right)\left(-\frac{1}{\sigma_{S}^{2}}, \lambda, \frac{1}{\sigma_{S}^{2}}\right), \\
& B_{\Psi, 2}^{\top} U_{2} A_{\Psi, 2}=o_{2}\left(\begin{array}{c}
-\frac{1}{\sigma_{S}^{2}} \\
\lambda \\
\frac{1}{\sigma_{S}^{2}}
\end{array}\right)\left(0, \frac{1}{o_{2}^{c}}-\frac{1}{\sigma_{\Theta}^{2} \mu_{2}^{2}}, 0\right) .
\end{aligned}
$$

Define $\kappa=\sigma_{2}\left(\frac{\sigma_{f}}{\sigma_{s}}+\lambda^{2} \sigma_{\Theta}^{2}+\frac{1}{\sigma_{s}^{2}}\right)$. The reader can verify that

$$
\Xi_{2}=\Sigma_{2}-\frac{o_{2}}{1+\kappa} N^{\top} N
$$


where $\Sigma_{2}=\operatorname{diag}\left(o_{1}, \sigma_{\Theta}^{2}, \sigma_{S}^{2}\right)$ and $\left.Y=1-\frac{\partial_{1}}{\sigma_{s}} ; \lambda \sigma_{\Theta}^{2}, \lambda\right)$. We thus nave

$$
\Xi_{2} B_{\Psi, 2}^{i} C_{2} A_{\Psi, 2}=\frac{o_{2}}{1+\kappa}\left(\begin{array}{c}
-\frac{1}{\sigma_{\xi}^{2}} \\
\lambda \sigma_{\theta}^{2} \\
1
\end{array}\right)\left(0, \frac{1}{o_{2}^{c}}-\frac{1}{\sigma_{\theta}^{2} \mu_{2}^{2}}, 0\right)
$$

Defining $h=B_{Q, 2} \overline{2}_{2} B_{Q, 2}^{\top}$ and noting that $B_{Q, 2}=\left(p_{n .2}-b_{2}\right)\left(1,-\mu_{2}, 0\right)$, we get

$$
h=\frac{\left(p \Pi .2+b_{2}\right)^{2} \mu_{2}^{2} \sigma_{\Theta}^{2} o_{1}}{o_{2}(1+\kappa)} \text {. }
$$

We can now conclude that $X_{1}^{i}=d_{\theta, 1} \hat{\Theta}_{1}^{i}+d_{\Delta, 1} \Delta_{i}^{i}$, where

$$
\begin{aligned}
& d_{\theta, 1}=\frac{1}{\lambda h} e_{\theta, 2}=\frac{1}{\lambda h} p_{\theta .1} \\
& d_{\Delta .1}=\frac{1}{\lambda h}\left(e_{\Pi .2}+\frac{o_{2}\left(p_{\Pi .2}+b_{2}\right)\left(\frac{o_{1}}{\sigma_{c}^{2}}+\lambda \mu_{2} \sigma_{\Theta}^{2}\right)\left(\frac{1}{\sigma_{2}^{2}}-\frac{1}{\gamma_{3}^{2} \mu_{2}^{2}}\right)}{1+\kappa}\right)
\end{aligned}
$$

where $e_{\Pi, 2}=p_{\Pi .2}+b_{2}-p \Pi, 1$ with $b_{2}=(1-p \Pi .2) \frac{o_{2}^{c}}{\mu^{2} \sigma_{\vartheta}^{2}}$. Imposing conditions on $d_{\Theta, 1}$ and $d_{\Delta, 1}$ as in (25), we obtain

$$
\begin{aligned}
& p_{\theta, 1}=\lambda h \\
& p_{\Pi, 1}=\frac{o_{1}^{\varepsilon}}{o_{1}^{c}+\sigma_{S}^{2}}\left(p \pi .2+b_{2}\right)\left(1+\frac{o_{2}\left(\frac{o_{1}}{\sigma_{S}^{2}}+\lambda \mu_{2} \sigma_{\theta}^{2}\right)\left(\frac{1}{o_{2}^{\varepsilon}}-\frac{1}{\sigma_{9}^{\frac{1}{2}} \mu_{2}^{2}}\right)}{1+\kappa}\right)
\end{aligned}
$$

where we recall

$$
p \Pi .2=\frac{o_{2}^{c}}{o_{2}^{c}+w_{2}}, \quad h=\frac{\left(p \Pi .2+b_{2}\right)^{2} \mu_{2}^{2} \sigma_{\ominus}^{2} o_{1}}{\left.o_{2}(1+\kappa)\right)}, \quad \mu_{2}=\frac{\lambda \sigma_{S}^{2}}{2} .
$$

The reader can verify that

$$
p_{\Pi .1}=\frac{o_{1}}{\sigma_{S}^{2}}\left(p_{\Pi .2}+b_{2}\right) \frac{1+o_{2}\left(\frac{2}{\sigma_{S}^{2}}+\frac{\lambda^{2} \sigma_{a}^{2}}{2}\left(1+\frac{\sigma_{S}^{2}}{o_{1}}\right)\right)}{1+\kappa}=\frac{o_{1}}{\sigma_{S}^{2}}\left(p_{\Pi .2}+b_{2}\right) \frac{1+\frac{\lambda^{2} \sigma_{g}^{2} \sigma_{S}^{2}}{2}}{1+\kappa}
$$

and

$$
\left(p \Pi .2+b_{2}\right) \mu_{2}^{2} \sigma_{\Theta}^{2}=o_{2}\left(1+\frac{\lambda^{2} \sigma_{\Theta}^{2} \sigma_{S}^{2}}{2}\right) .
$$

Thus,

$$
\mu_{1}=\lambda \frac{\left(p \Pi .2+b_{2}\right) \mu_{2}^{2} \sigma_{\Theta}^{2} \sigma_{S}^{2}}{\sigma_{2}\left(1+\frac{\lambda^{2} \sigma_{Q}^{2} \sigma_{S}^{2}}{2}\right)}=\lambda \sigma_{S}^{2}=\lambda w_{1}
$$

This completes our proof. 


\section{Footnotes}

1. Although we do not focus on the behavior of price volatility here, it is worth pointing out that under differential information there are subsequent price changes after an exogenous information shock [see also Grundy and McNichols (1989), and Shalen (1993)]. This implies that with private information, independent information flow can, over time, generate serially correlated changes in prices. However, the subsequent price changes are small compared with the initial response, and decrease over time. The corresponding volume can be comparable to the initial volume and vary a lot.

2. See, e.g., Gallant, Rossi and Tauchen (1992), Campbell, Grossman, and Wang (1993), and LeBaron (1992) for results on the aggregate stock trading volume.

3. For models of dynamic trading without private information, see Huffman (1987) and Dumas (1989). See also Wang (1994).

4. Kyle $(1985,1989)$ considers the situation when informed investors behave strategically maximize the gains of their information trading. They trade gradually in order to prevent private information from being revealed too quickly and to extract more profits. Admati and Pfleiderer (1988) also study a trading model with strategic behavior. In this paper, we follow the competitive approach.

5. Singleton (1987) considers the special case when private information is short-lived - the true state of the economy is revealed after two periods. In the current model, we do allow long-lived private information - the true state of the economy may never be revealed.

6. We note that in models with three dates $(0,1,2)$, trading occurs only in the first two dates. The volume on the first date depends on the specification of investors' initial endowments. The only truly endogenous volume is the volume in the second date. Thus, these models cannot be used to analyze the dynamics of volume. Shalen (1993) analyzes the volume in a setting with three dates.

7. The more general situation would be to allow $\epsilon_{S, t}^{i}$ to have different variance for different investors, and to be correlated across investors. Let $i$ and $i^{\prime}$ be two investors. In the case that $\operatorname{Var}\left[\epsilon_{S, t}^{i}\right]<\operatorname{Var}\left[\epsilon_{S, \ell}^{i^{\prime}}\right]$ and $E\left[\epsilon_{S, \ell}^{i^{\prime}} \epsilon_{S, \ell}^{i}\right]=0$, investor $i$ has a signal independent from that of $i^{\prime}$ but with better precision. However, since the two signals are independent, $\epsilon_{S, \ell}^{i^{\prime}}$ is still informative to $i$ given $\epsilon_{S, t}^{i}$. If $\mathbf{E}\left[\epsilon_{S, t}^{i^{\prime}} \epsilon_{S, t}^{i}\right] \neq 0$, there would be common noise in the two investors' signals. 
Suppose there is common noise in all the investors' signals. Then, aggregation of information across all investors would not reveal the true value of $\Pi$ even when the number of investors goes to infinity [see Pfleiderer (1984)]. Another case is when $\epsilon_{S, t}^{i^{\prime}}=\epsilon_{S, t}^{i}+e_{t}$ and $\mathrm{E}\left[e_{t} \mid \epsilon_{S, t}^{i}\right]=0$. Then, the signal $\epsilon_{S, t}^{i}$ strictly dominates the signal $\epsilon_{S, t}^{i^{\prime}}$. Given $\epsilon_{S, t}^{i}$, no additional information is provided by $\epsilon_{S, \ell}^{i^{\prime}}$. In this case, investor $i$ has superior information than $i^{\prime}$. For this paper, we restrict ourselves to the case with $\epsilon_{S, t}^{i}$ i.i.d. across all investors.

8. The combination of CARA preferences and normally distributed payoffs is often used in the literature to study the linear rational expectations equilibrium under asymmetric information. See, e.g., Grossman (1976, 1981), Grossman and Stiglitz (1980), Hellwig (1980), and Diamond and Verrecchia (1981) in static settings, and Grundy and McNichols (1989), Brown and Jennings (1989), and Wang (1994) in dynamic settings.

9. If the noises in private signals are correlated, the average of the signals does not give the true value of $\Pi$. Instead, it will be $\Pi$ plus the common noise. Our analysis can be extended to this case.

10. Due to the symmetry among investors in the quality of their signals, their signals should enter the price function with equal weights. Thus, the general form of the price function satisfying the symmetry will be

$$
P_{t}=\sum_{s=0}^{t}\left[b_{t, s}^{s} \int_{i} S_{t-s}^{i}+b_{t, s}^{Y} Y_{t-s}-b_{t, s}^{\theta} \Theta_{t-s}\right] \text {. }
$$

Since $\int_{i} S_{t-s}^{i}=\Pi$ by the Law of Large Numbers, we arrive at the form in (12).

11. At $t=1, P_{1}=\mathrm{L}\left[\Pi, \Theta_{1}, Y_{1}\right]$. Thus, $\Theta_{1}=\mathrm{L}\left[\Pi, P_{1}, Y_{1}\right]$. At $t=2, P_{2}=\mathrm{L}\left[\Pi, \Theta_{1}, \Theta_{2}, Y_{1}, Y_{2}\right]=$ $\mathbf{L}\left[\Pi, \Theta_{2}, Y_{2}, P_{1}, Y_{1}\right]$. The statement then follows by induction.

12. Clearly, $\left\{\hat{\Pi}_{t}^{i}, \hat{\Theta}_{i}^{i}\right\}$ follows a Gaussian Markov process under $\mathcal{F}_{t}^{i}$. In order to see that $\left\{\hat{\Pi}_{t}^{i}, \hat{\Theta}_{t}^{i}, \hat{\Pi}_{t}^{c}, \hat{\Theta}_{t}^{c}\right\}$ does as well, note that we can rewrite (15) as

$$
\left(\begin{array}{c}
\hat{\Pi}_{t}^{c} \\
\hat{\Theta}_{t}^{c}
\end{array}\right)=\left(\begin{array}{c}
\mathbf{E}_{t-1}^{c}[\Pi] \\
\mathbf{E}_{t-1}^{c}\left[\Theta_{t}\right]
\end{array}\right)+K_{t}^{c}\left(\begin{array}{c}
\mathbf{E}_{t-1}^{i}\left[\xi_{t}\right]-\mathbf{E}_{t-1}^{c}\left[\xi_{t}\right] \\
\mathbf{E}_{t-1}^{i}\left[Y_{t}\right]-\mathbf{E}_{t-1}^{c}\left[Y_{t}\right]
\end{array}\right)+K_{t}^{c}\left(\begin{array}{c}
\xi_{t}-\mathbf{E}_{t-1}^{i}\left[\xi_{t}\right] \\
Y_{t}-\mathbf{E}_{t-1}^{i}\left[Y_{t}\right]
\end{array}\right) .
$$

Furthermore, $\mathbf{E}_{t-1}^{c}\left[\xi_{t}\right]=\hat{\Pi}_{t-1}^{c}-a_{\theta} \mu_{t} \hat{\Theta}_{t-1}^{c}, E_{t-1}^{i}\left[\xi_{t}\right]=\hat{\Pi}_{t-1}^{i}-a_{\theta} \mu_{t} \hat{\Theta}_{t-1}^{i}, E_{t-1}^{c}\left[Y_{t}\right]=\hat{\Pi}_{t-1}^{c}$, $\mathbf{E}_{t-1}^{i}\left[Y_{t}\right]=\hat{\Pi}_{t-1}^{i}$ and $\left\{\hat{\Pi}_{t}^{i}, \hat{\Theta}_{t}^{i}, \hat{\Pi}_{t}^{c}, \hat{\Theta}_{t}^{c}\right\} \subseteq \mathcal{F}_{t}^{i}$. Thus, $\left\{\hat{\Pi}_{t}^{i}, \hat{\Theta}_{t}^{i}, \hat{\Pi}_{t}^{c}, \hat{\Theta}_{t}^{c}\right\}$ follows a Markov process under $\mathcal{F}_{\mathfrak{t}}$.

13. Note that the Grundy-McNichols model corresponds to the case where $\sigma_{\delta} \neq 0$. In their model, there are common noises in investors' signals. The common noise gives the residual risk 
in the stock's payoff that is resolved only at the terminal date. The collection of all investors' private information does not fully reveal the true value of the stock. When the common noise goes to zero, their model reduces to our model with $\sigma_{\delta}=0$.

14. See, e.g., Grossman and Miller (1988), Campbell and Kyle (1993), Wang (1993) and Spiegel and Subrahmanyam (1992) for more detailed discussions on the equilibrium price in settings similar to this.

15. Here, the expectation is taken with respect to the unconditional distribution. One way to justify this is to assume that the prior is just the stationary distribution as we did in Section 1. This gives $\operatorname{Var}\left(\Delta \theta_{t}\right)=\frac{2 \sigma_{\theta}^{2}}{1+a_{\theta}}$.

16. See also eq.(24), which shows that on $T-1$, the date before the terminal date, $p_{\Pi, T-1}=1-\alpha_{t}$. This is the static result.

17. Exactly speaking, $X_{i}^{i}$ is a linear combination of two parts, one corresponding to the growth part of the stock demand that is proportional to the expected stock returns, and the other corresponding to the hedging part given that the expected return changes over time [see Merton (1971)]. Since the expected return is a linear function of the two state variables $\hat{\Theta}_{t}^{i}$ and $\Delta_{t}^{i}$ and so is the holding of the hedging portfolio, we can express the total holding as a linear function of the two state variables. Because $\Delta_{i}^{i}$ is non-zero only when investor $i$ has private information, we term as speculative the part of his stock holding that depends on $\Delta_{i}^{i}$.

18. It is also possible that investors on the two sides of a trade both think that their trades are non-informational, but the trading is purely due to differential information. Consider the following example: Suppose that from $t-1$ to $t$, investors maintain their speculative positions and there is no change the actual stock supply. However, half of the investors think that the supply has increased by $10 \%$ (i.e., $\Delta \hat{\theta}_{t}^{i}=0.1$ ), while the other half thinks that the supply has decreased by $10 \%$ (i.e., $\Delta \hat{\Theta}_{t}^{i^{\prime}}=0.1$ ). Then there will be trading between the two groups (with 0.1 volume) and all investors think that their own trades are non-informational.

19. Although the decomposition of $X_{t}^{i}$ into $\theta_{t}$ and $x_{t}^{i}$ has its intuitive appeal, it is based on the information of the true values of the underlying state variables $\Phi_{t}$ (which only God sees). Thus, $x_{i}^{i}$ has no clear economic interpretation at any individual level, since it is not measurable to any investor's information set $\mathcal{F}_{i}$. Furthermore, such a decomposition relies crucially on the fact that non-informational trading is exogenous. If the non-informational trading is endogenous, it will be intimately related to the informational trading. There would be no simple dichotomy between the two as we have here. See Wang (1994) for a discussion of this issue in a different setting. 
20. See Pfleiderer (1984) for a discussion on trading volume when there are supply shocks and a countable number of traders.

21. Suppose that one solves a one-period equilibrium at $t$, assuming that investor $i$ has information $\mathcal{F}_{t}^{i}$, and the risky asset will be liquidated at $t+1$. Then, $p_{\Pi, t}=1-\alpha_{t}, p_{\theta, t}=\lambda o_{t}$, and the optimal stock holding of investor $i$ is $X_{t}^{i}=\hat{\theta}_{t}^{i}+\frac{\alpha_{l}}{\lambda_{o_{t}}} \Delta_{i}^{i}$. It is easy to verify that the coefficients of the equilibrium price in this repeated one-period equilibrium satisfy $\mu_{t}=\lambda w_{t}$, as in Corollary 2. Moreover, since $\frac{\alpha_{l}}{\lambda_{a_{l}}}=\frac{\alpha_{l}}{\mu_{t}\left(1-\alpha_{t}\right)}$, the optimal stock holding of investor $i$ in the repeated one-period model is exactly the same as that in the multi-period model.

22. This is best seen by looking at investors' positions in the stock. Given that $w_{t}=w_{1}$ for $t=1, \cdots, T-1$, then $\mu_{t}=\lambda w_{t}=\mu_{1}$ and from (27)

$$
x_{t}^{i}=\frac{1}{\mu_{t}}\left(\hat{\Pi}_{t}^{p, i}-\Pi\right)=\frac{1}{\mu_{1}}\left(S_{1}^{i}-\Pi\right)
$$

which is constant for $t>1$. Thus, the volume of informational trading is zero after the first period.

23. Various volume patterns can be found in the strategic trading models by Foster and Viswanathan (1993a), Holden and Subrahmanyam (1992), and Vayanos (1992). Howeve:, differential information is not a major issue in these models, although Vayanos's model has some fiavor of differential information, since the investors' initial endowment is not known to each other. A more recent paper by Foster and Viswanathan (1993b) considers the situation of differential information in a strategic setting.

24. Grundy and McNichols (1989), Kim and Verrecchia (1991a,b), and Wang (1994) analyze the link between volume on announcement days and heterogeneous information. See also Stickel and Verrecchia (1993).

25. In a partial equilibrium model with no differential information, we may expect investors to trade less aggressively prior to the announcement date, (due to their aversion towards risk). Here, we are in a general equilibrium model with differential information. Investors may speculate more aggressively on the outcome of the announcement despite the risk involved. Note that in the current setting investors in general do not have concordant expectations and the "No-Trade Theorem" [see, e.g., Milgrom and Stokey (1982)] does not apply. In equilibrium, investors do have different expectations and interpretations about public information, and they trade on it. Grundy and McNichols (1989) give a detailed discussion on this issue in a simpler setting. 
26. It is possible (for some parameter values) that the volatility at the date right before the announcement is even higher than the volatility at the announcement date. 


\section{References}

Admati, A., 1985, “A Noisy Rational Expectations Equilibrium for Multi-Asset Securities Markets," Econometrica, 53, 629-657.

Admati, A., and P. Pfleiderer, 1988, "A Theory of Intraday Patterns: Volume and Price Variability," Review of Financial Studies, 1, 3-40.

Brown, D.P., and R.H. Jennings, 1989, "On Technical Analysis," Review of Financial Studies, 2, 527-552.

Campbell, J.Y., S.J. Grossman and J. Wang, 1993, "Trading Volume and Serial Correlation in Stock Returns," Quarterly Journal of Economics, 108, 905-939.

Campbell, J.Y., and A.S. Kyle, 1993, "Smart Money, Noise Trading, and Stock Price Behavior," Review of Economic Studies, 60, 1-34.

Clark, P.K., 1973, "A Subordinate Stochastic Process Model with Finite Variance for Speculative Prices," Econometrica, 41, 135-155.

Diamond, D.W., and R.E. Verrecchia, 1981, "Information Aggregation in a Noisy Rational Expectations Economy," Journal of Financial Economics, 9, 221-235.

Dumas, B., 1989, "Two-Person Dynamic Equilibrium in the Capital Market," Review of Financial Studies, 2, 157-188.

Feldman, M., and C. Gilles, 1985, "An Expository Note on Individual Risk without Aggregate Uncertainty," Journal of Economic Theory, 35, 26-32.

Foster, F.D., and S. Viswanathan, 1993a, "Strategic Trading with Asymmetrically Informed Traders and Long-Lived Information," working paper, Duke University.

Foster, F.D., and S. Viswanathan, 1993b, "Strategic Trading when Agents Forecast the Forecasts of Others," working paper, Duke University.

Gallant, A.R., P.E. Rossi and G. Tauchen, 1992, "Stock Prices and Volume," Review of Financial Studies, 5, 199-242.

Gennotte, G., and A.S. Kyle, 1991, "Intertemporal Insider Trading with a Smooth Order Flow," working paper, University of California at Berkeley. 
Grossman, S.J., 1976, "On the Efficiency of Competitive Stock Markets where Traders Have Diverse Information," Journal of Finance, 31, 573-585.

Grossman, S.J., 1981, "An Introduction to the Theory of Rational Expectations Under Asymmetric Information," Review of Economic Studies, 48, 541-559.

Grossman, S.J., and M.H. Miller, 1988, "Liquidity and Market Structure," Journal of Finance, 43, 617-633.

Grossman, S.J., and J. Stiglitz, 1980, "On the Impossibility of Informationally Efficient Markets," American Economic Review, 70, 383-408.

Grundy, B., and M. McNichols, 1989, "Trade and Revelation of Information through Prices and Direct Disclosure," Review of Financial Studies, 2, 495-526.

Hellwig, M.F., 1980, "On the Aggregation of Information in Competitive Markets," Journal of Economic Theory, 22, 477-498.

Holden, C., and A. Subrahmanyam, 1990, "Long-Lived Private Information and Imperfect Competition," Journal of Finance, 47, 247-270.

Huffman, G.W., 1987, "A Dynamic Equilibrium Model of Asset Prices and Transactions Volume," Journal of Political Economy, 95, 138-159.

LeBaron, B., 1992, "Persistence of the Dow Jones Index on Rising Volume," working paper, University of Wisconsin.

Kim, O., and R.E. Verrecchia, 1991a, "Trading Volume and Price Reactions to Public Announcements," Journal of Accounting Research, 29, 302-321.

Kim, O., and R.E. Verrecchia, 1991b, "Market Reaction to Anticipated Announcements," Journal of Financial Economics, 30, 273-309.

Kyle, A.S., 1985, "Continuous Auctions and Insider Trading," Econometrica, 53, 1315-1335.

Kyle, A.S., 1989, "Informed Speculation with Monopolistic Competition," Review of Economic Studies, 56, 317-355.

Lamoureux, C.G., and W.D. Lastrapes, 1990, "Heteroskedasticity in Stock Return Data: Volume vs. GARCH Effects," Journal of Finance, 45, 487-498. 
Lamoureux, C.G., and W.D. Lastrapes, 1992, "Endogenous Trading Volume and Momentum in Stock Return Volatility," working paper, Washington University.

Liptser, R.S., and A.N. Shiryayev, 1974, Statistics of Random Processes I \& II, Springer-Verlag, New York.

Milgrom, P., and N. Stokey, 1982, "Information, Trade and Common Knowledge," Journal of Economic Theory, 26, 17-27.

Pfleiderer, P., 1984, "The Volume of Trade and the Variability of Prices: A Framework for Analysis in Noisy Rational Expectations Equilibria," working paper, Stanford University.

Rao, K.P.S.B., and M.B. Rao, Theory of Charges, Academic Press, New York.

Shalen, C.T., 1993, "Volume, Volatility, and the Dispersion of Beliefs," Review of Financial Studies, 6, 405-434.

Singleton, K.J., 1987, “Asset Prices in a Time-Series Model with Disparately Informed, Competitive Traders," New Approach to Monetary Economics (Proceedings of the Second International Symposium in Economic Theory and Econometrics), edited by W.A. Barnett and K.J. Singleton, Cambridge University Press.

Spiegel, M. and A. Subrahmanyam, 1992, "On Intraday Risk Premia," working paper, Columbia University.

Stickel, S.E. and R.E. Verrecchia, 1993, "Evidence that Volume Sustains Price Changes," working paper, University of Pennsylvania.

Townsend, R.M., 1983, "Forecasting the Forecasting of Others," Journal of Political Economy, $91,546-588$.

Vayanos, D., 1992, "A Dynamic Model of an Imperfectly Competitive Bid-Ask Market," working paper, MIT.

Wang, J., 1993, "A Model of Intertemporal Asset Prices under Asymmetric Information," Review of Economic Studies, 60, 249-282.

Wang, J., 1994, "A Model of Competitive Stock Trading Volume," Journal of Political Economy, $102,127-168$. 
$\overline{V_{t}}$

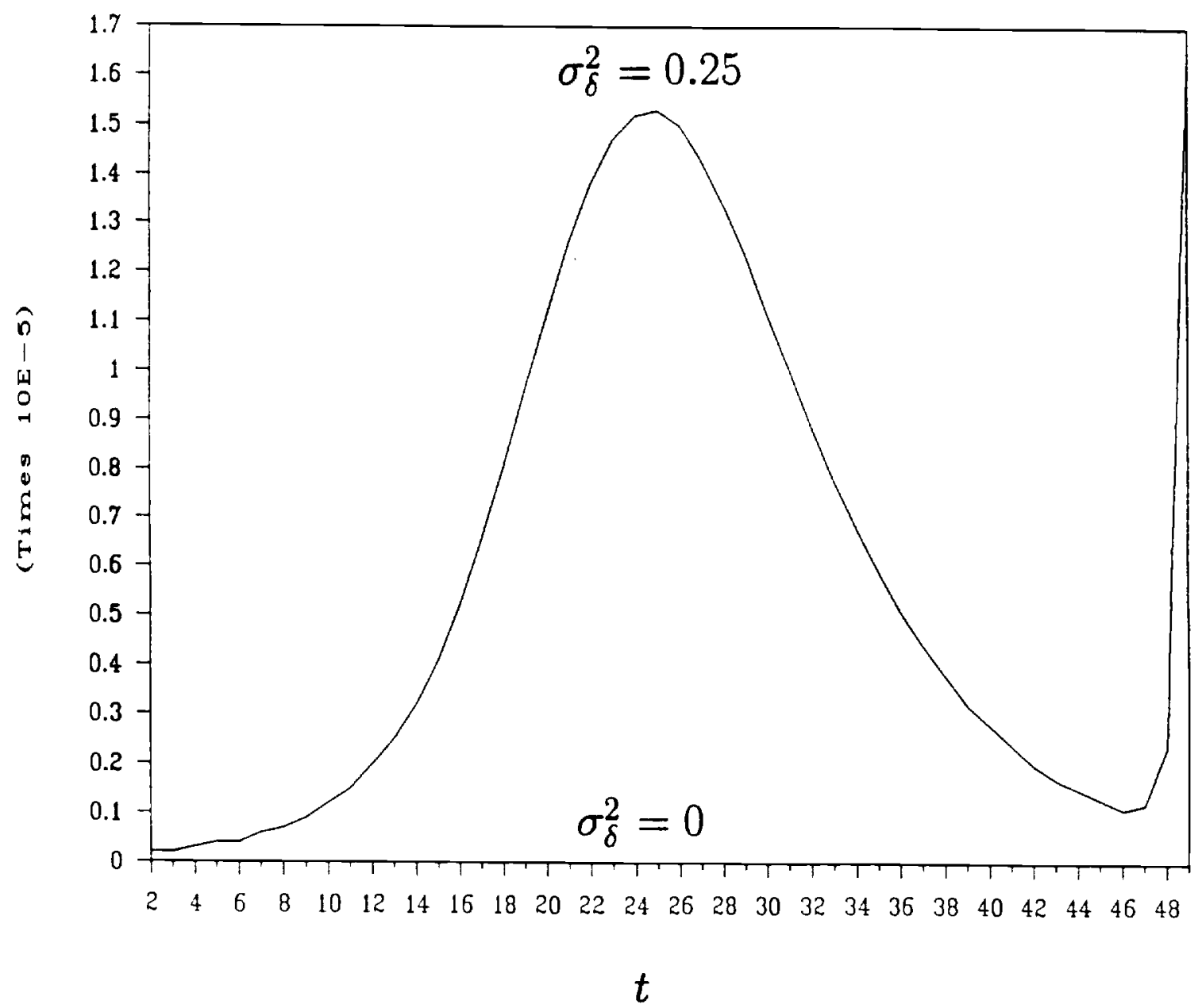


$h_{t}$

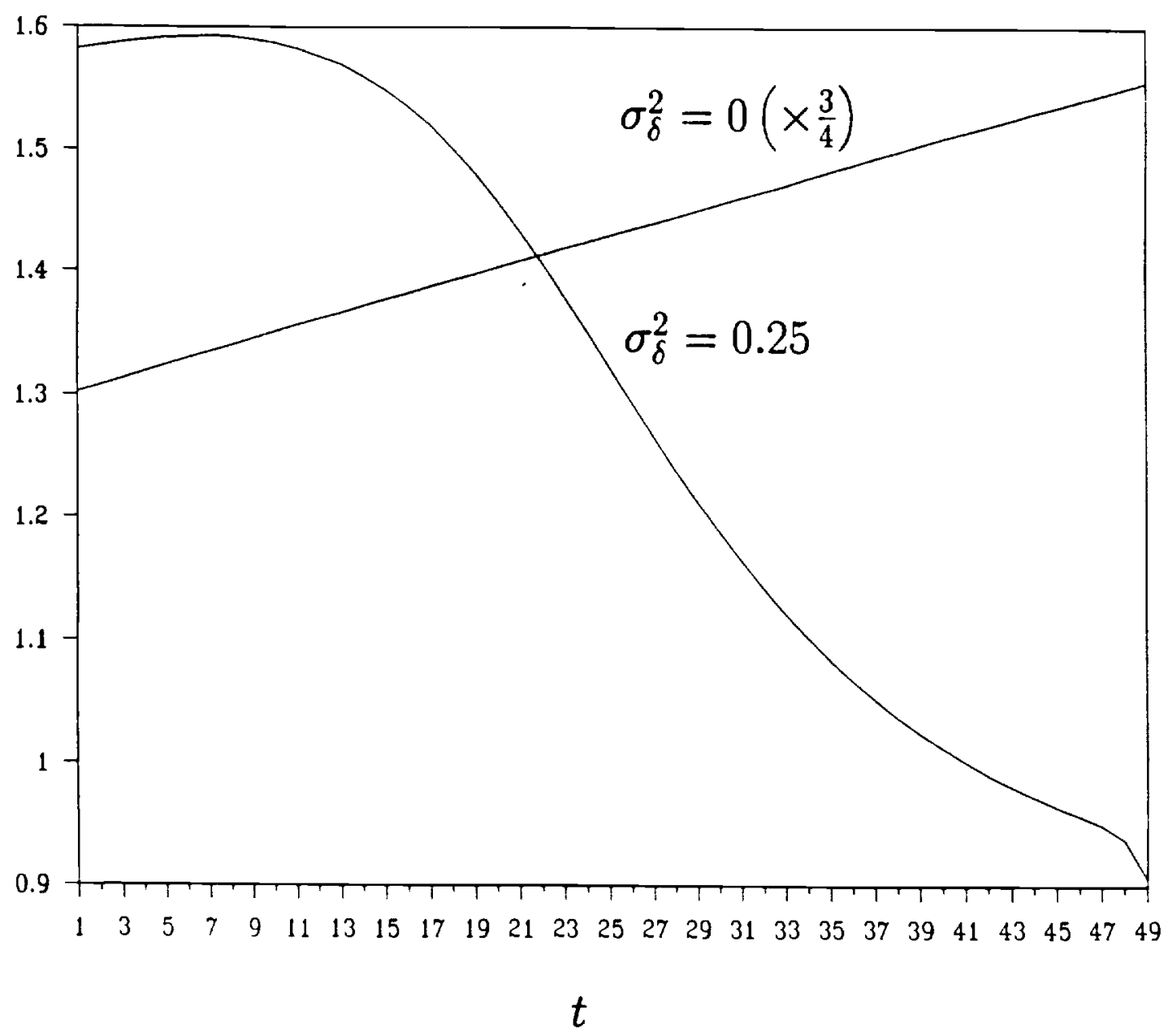


$\overline{\left|x_{t}^{i}\right|}$

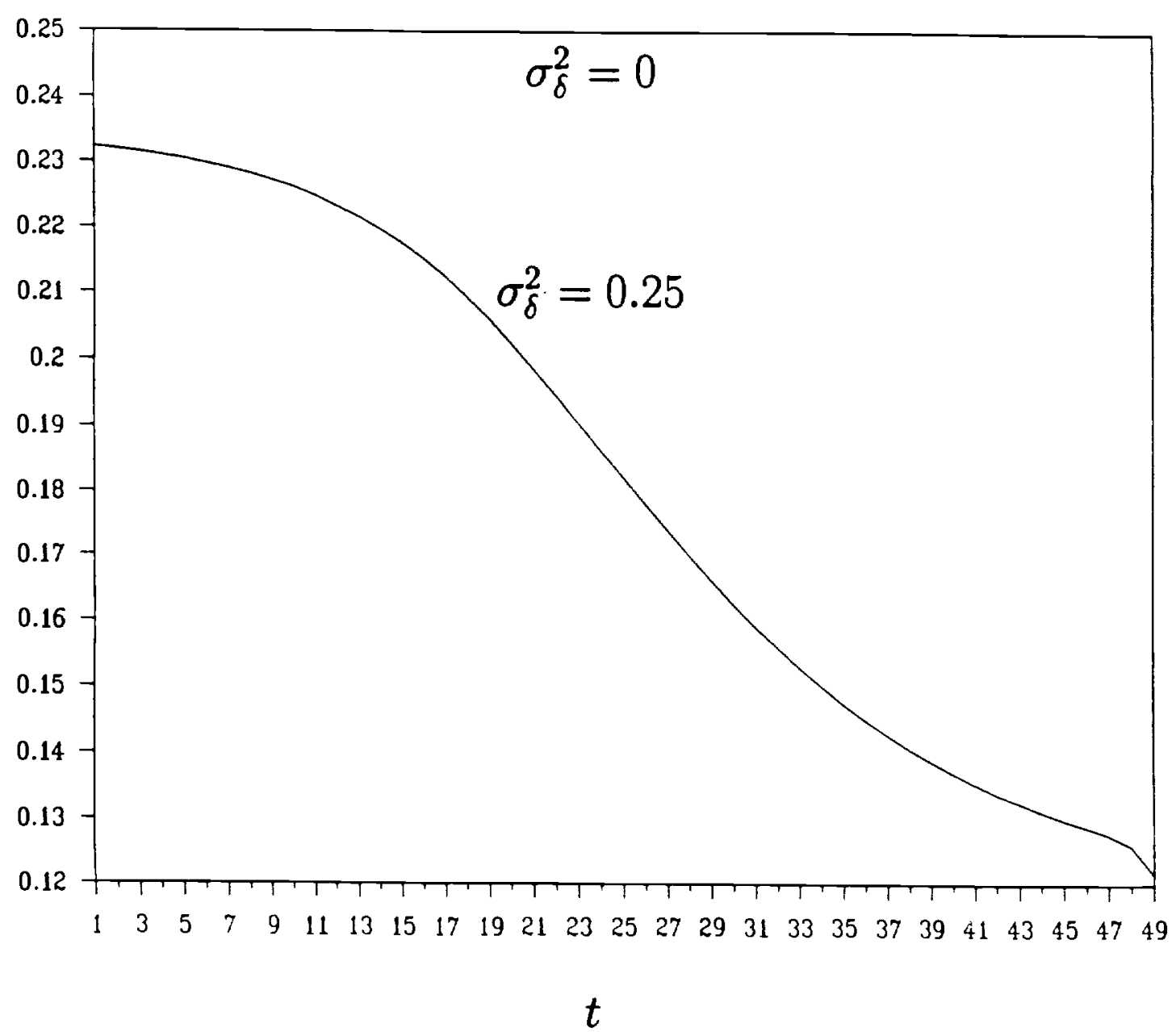


$\overline{V_{t}}$

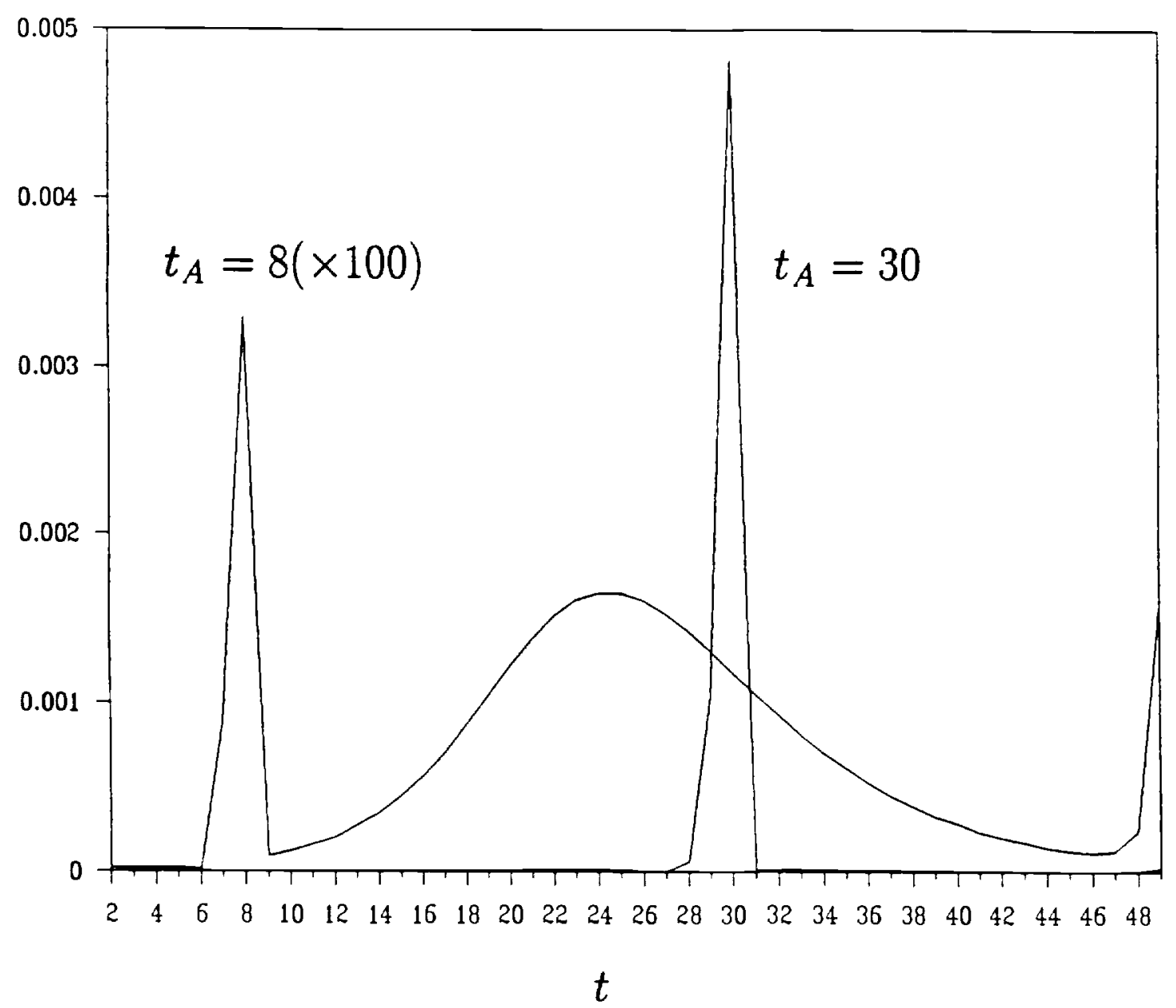


$h_{t}$

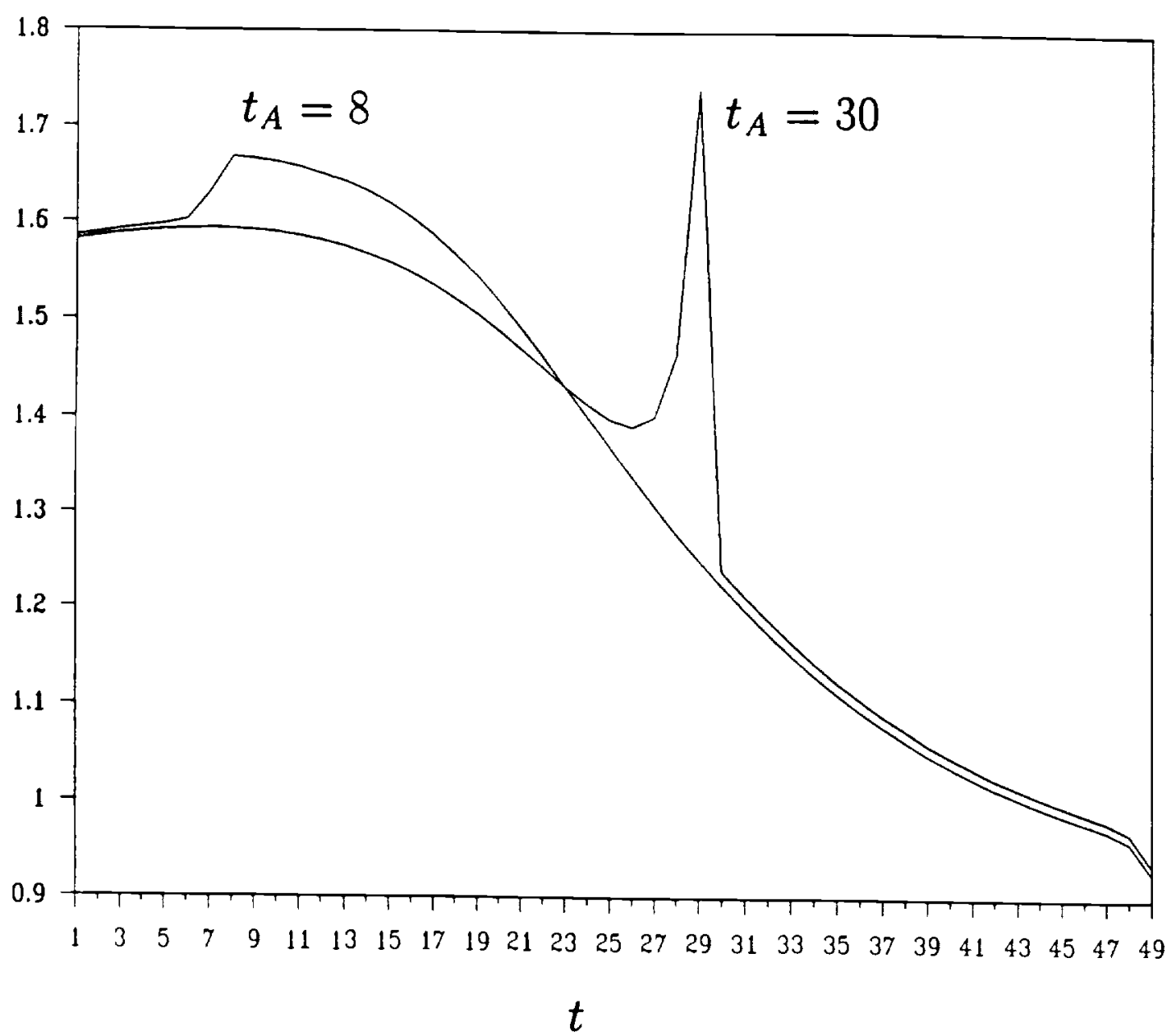




\section{$\overline{\left|x_{t}^{i}\right|}$}

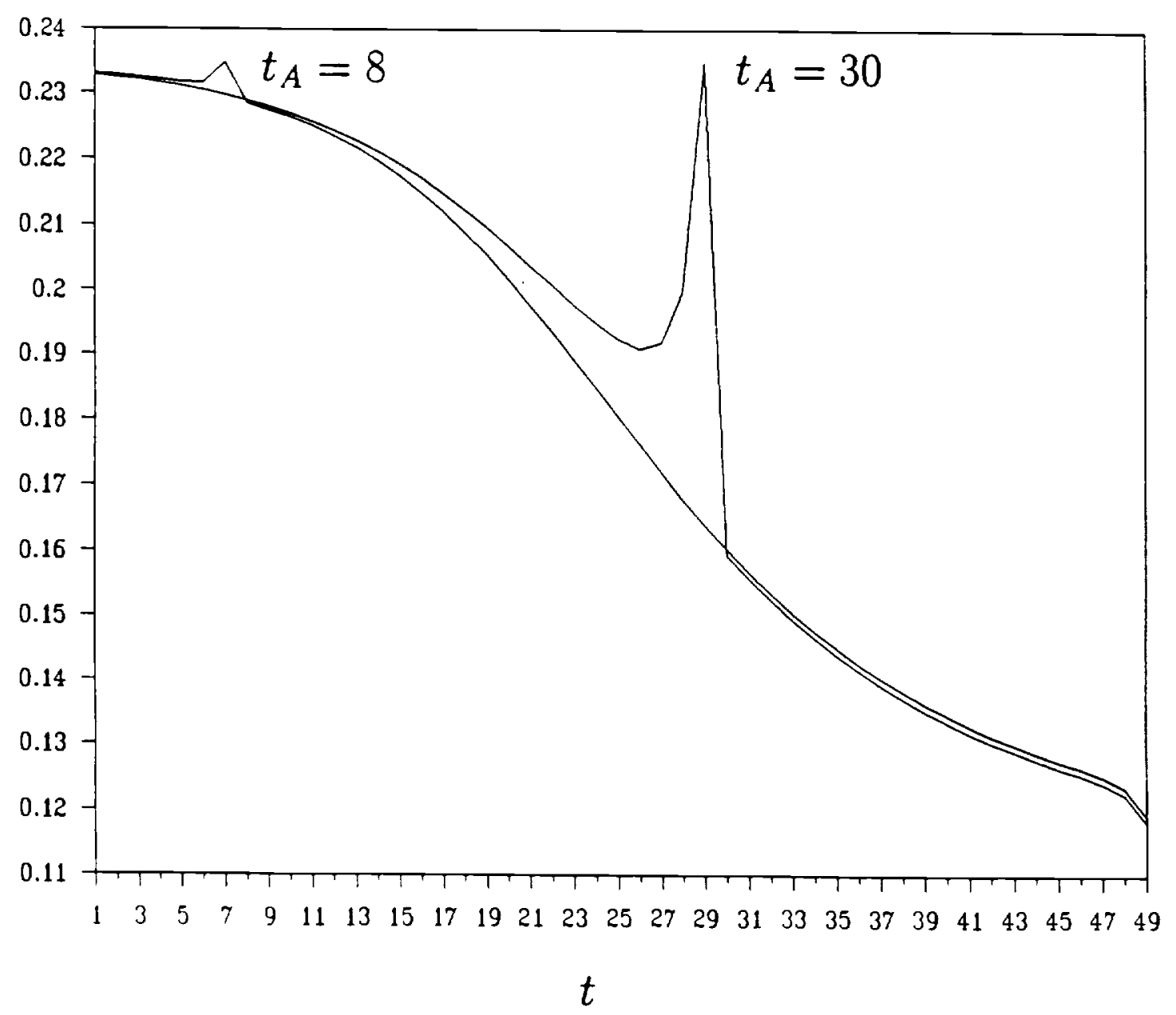


$p_{\Theta, t}$

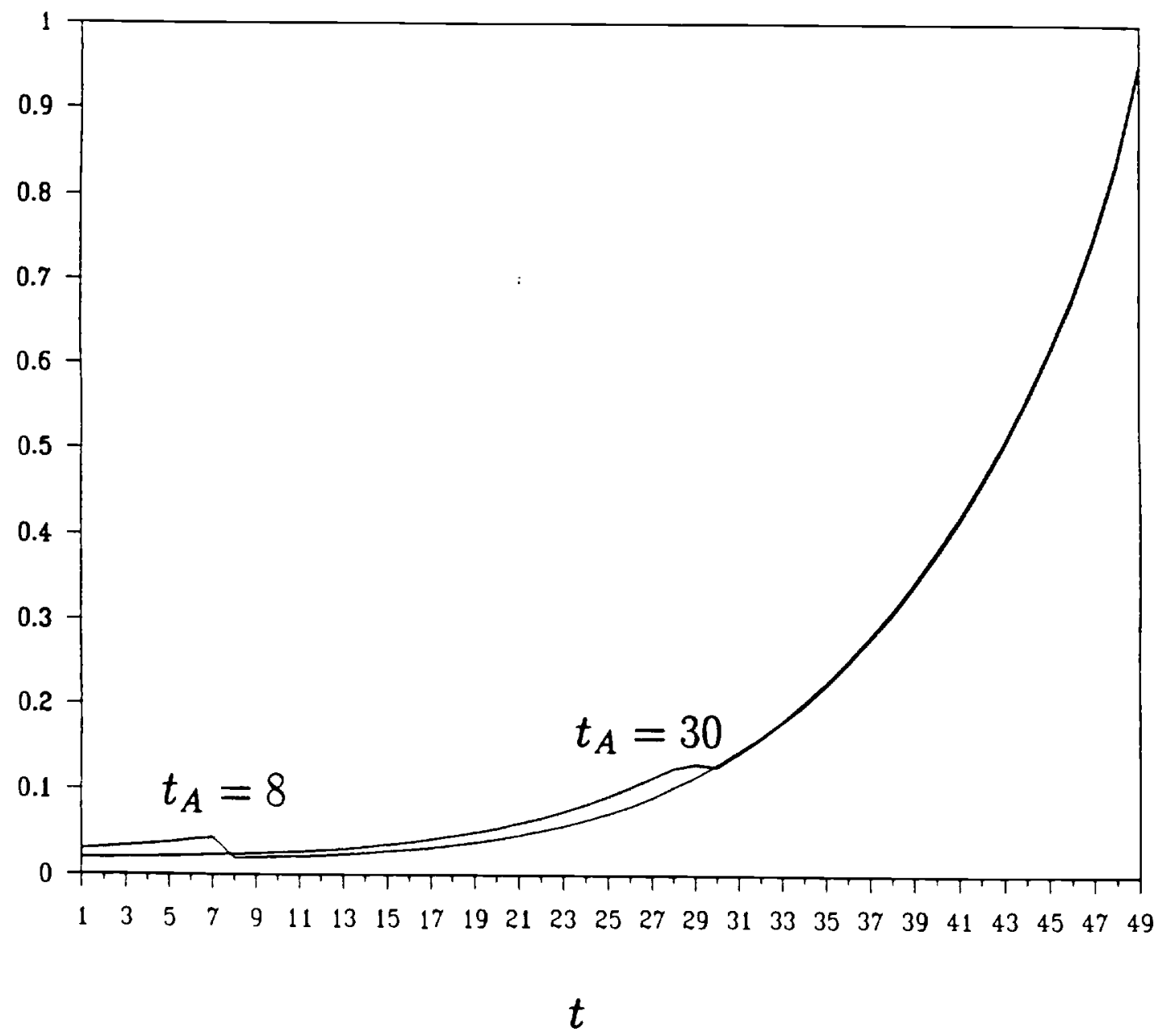


$p_{\Pi, t}$

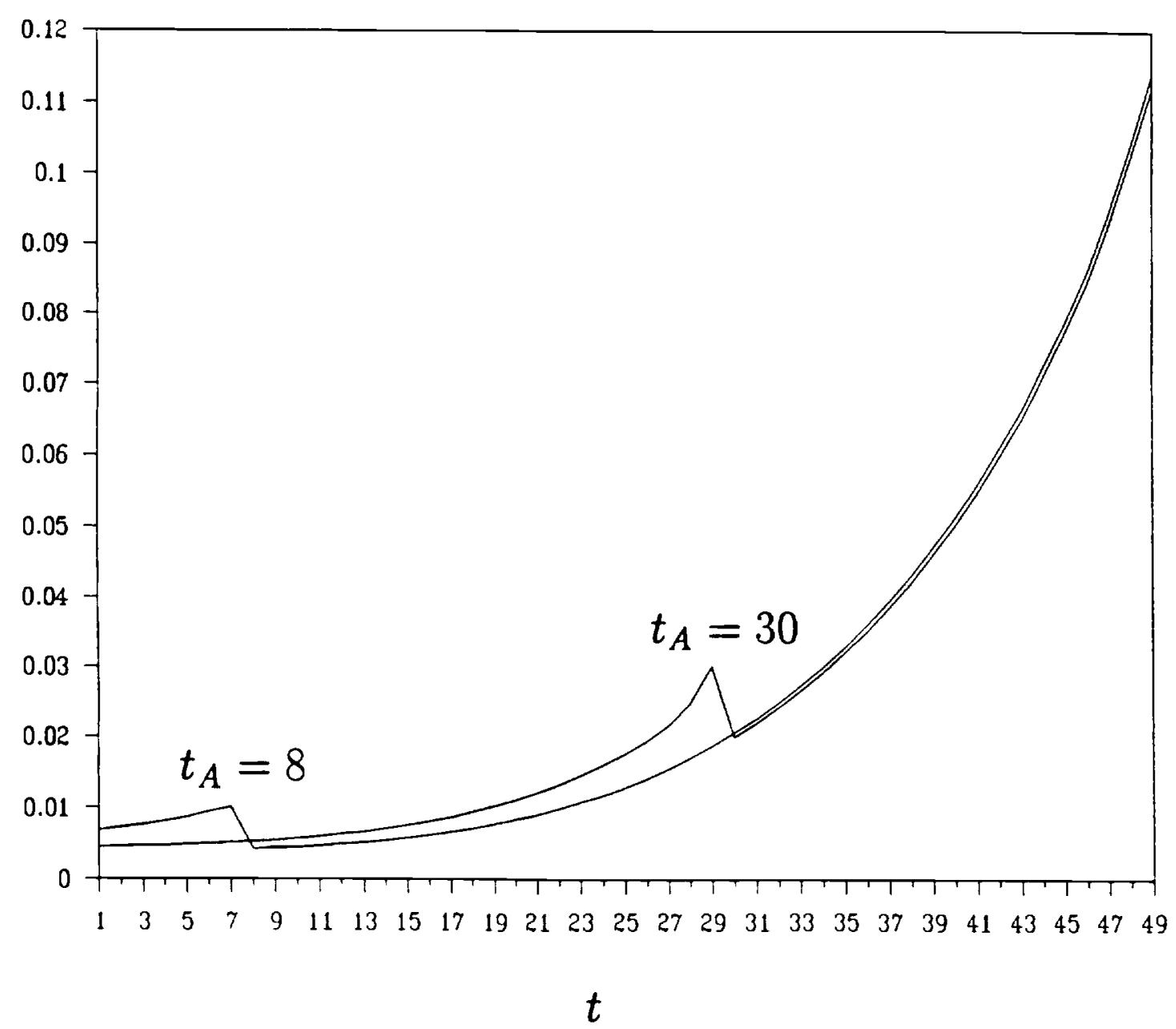


$o_{t}^{c}$

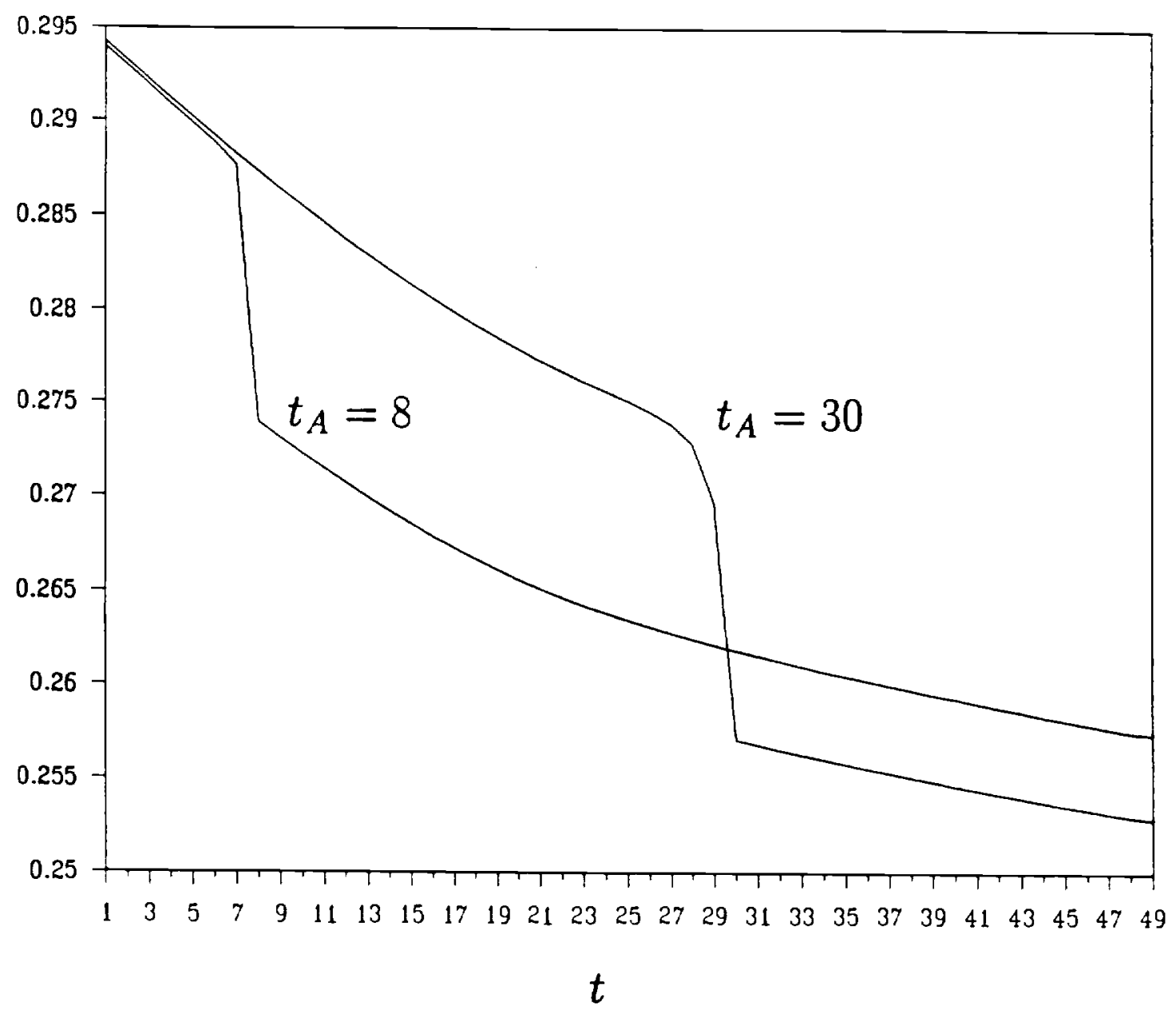


$\operatorname{Var}\left[P_{t}-P_{t-1}\right]$

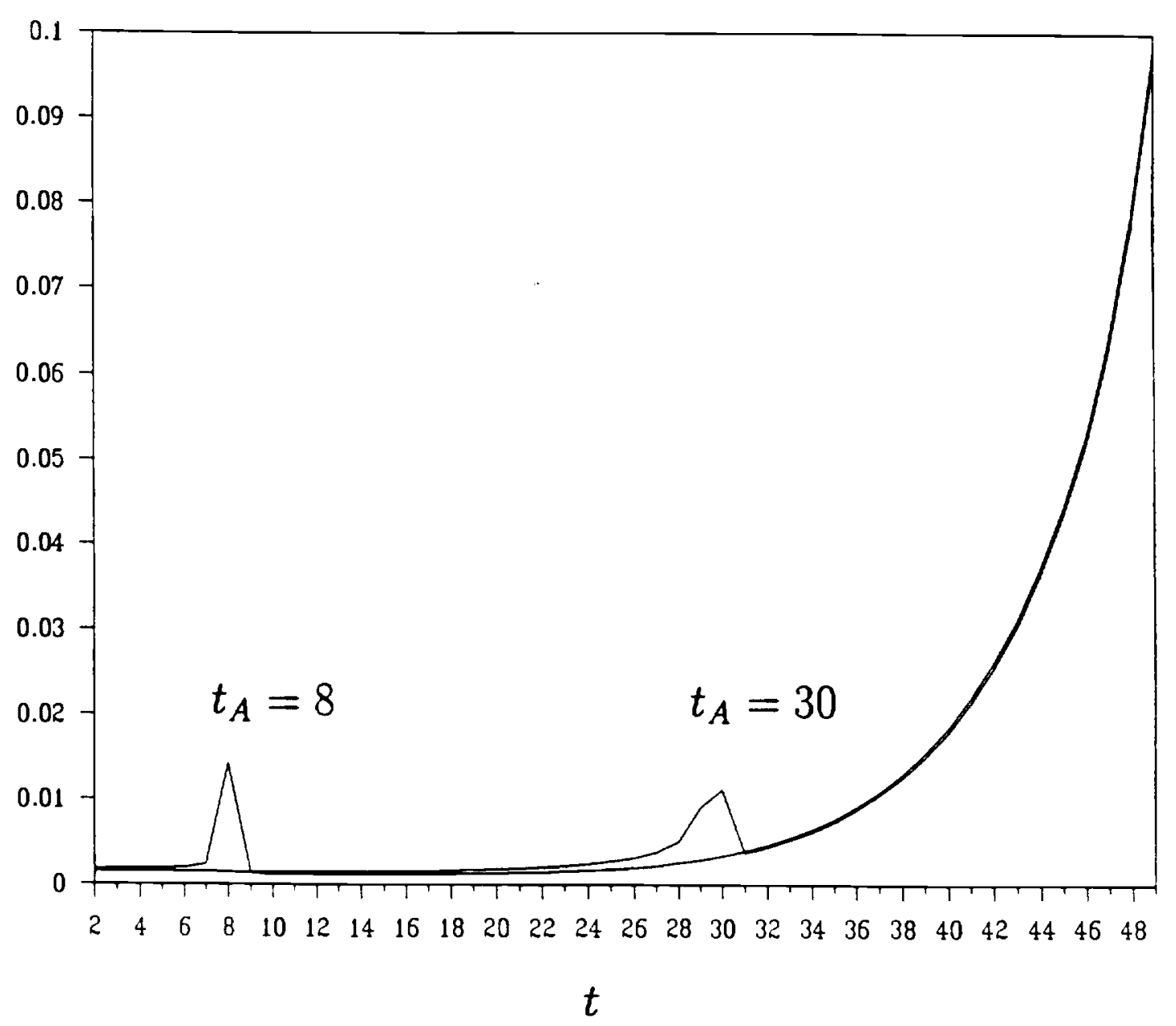


Figure 1. Expected volume of informational trading when there is no public announcement.

This figure plots the expected volume of informational trading $\bar{V}_{t}$ over time in the absence of public announcements. The parameters are set at the following values: $T=50, \lambda=2.0$, $a_{\theta}=0.85, \sigma_{n}^{2}=0.35, \sigma_{\Theta}^{2}=0.1, \sigma_{S, 1}^{2}=2.0, \sigma_{S, t}^{2}=10^{6}(1<t \leq T-1), \sigma_{Y, t}^{2}=10^{6}$ $(1 \leq t \leq T-1)$.

Figure 2. Intensity of investors' speculative trading when there is no public announcement.

This figure plots the intensity of investors' speculative trading $h_{t}$ over time in the absence of public announcements. The parameters are set at the following values: $T=50$, $\lambda=2.0, a_{\theta}=0.85, \sigma_{\Pi}^{2}=0.35, \sigma_{\theta}^{2}=0.1, \sigma_{S, 1}^{2}=2.0, \sigma_{S, t}^{2}=10^{6}(1<t \leq T-1), \sigma_{Y, t}^{2}=10^{6}$ $(1 \leq t \leq T-1)$.

Figure 3. Expected position of investors (net of supply shock) when there is no public announcement.

This figure plots investors' expected stock position (net of supply shock) $\overline{\left|X_{t}^{i}\right|}$ over time. The parameters are set at the following values: $T=50, \lambda=2.0, a_{\Theta}=0.85, \sigma_{\Pi}^{2}=0.35$, $\sigma_{\Theta}^{2}=0.1, \sigma_{S, 1}^{2}=2.0, \sigma_{S, t}^{2}=10^{6}(1<t \leq T-1), \sigma_{Y, t}^{2}=10^{6}(1 \leq t \leq T-1)$.

Figure 4. Expected volume of informational trading when there is a public announcement.

This figure plots the expected volume of informational trading $\bar{V}_{t}$ over time when there is a public announcement. The parameters are set at the following values: $T=50, \lambda=2.0$, $a_{\theta}=0.85, \sigma_{n}^{2}=0.35, \sigma_{\theta}^{2}=0.1, \sigma_{\delta}^{2}=0.25, \sigma_{S, 1}^{2}=2.0, \sigma_{S, t}^{2}=10^{6}(1<t \leq T-1)$, $\sigma_{Y, t_{A}}^{2}=6.0, \sigma_{Y, t}^{2}=10^{6}\left(t \neq t_{A}\right)$. The announcement dates are, respectively, $t_{A}=8$ and $t_{A}=30$.

Figure 5. Intensity of investors speculative trading when there is a public 


\section{announcement.}

This figure plots the intensity of investors speculative trading $h_{t}$ over time when there is a public announcement. The parameters are set at the following values: $T=50, \lambda=2.0$, $a_{\Theta}=0.85, \sigma_{\Pi}^{2}=0.35, \sigma_{\Theta}^{2}=0.1, \sigma_{\delta}^{2}=0.25, \sigma_{S, 1}^{2}=2.0, \sigma_{S, t}^{2}=10^{6}(1<t \leq T-1)$, $\sigma_{Y, t_{A}}^{2}=6.0, \sigma_{Y, t}^{2}=10^{6}\left(t \neq t_{A}\right)$. The announcement dates are, respectively, $t_{A}=8$ and $t_{A}=30$.

Figure 6. Expected position of investors (net of supply shock) when there is a public announcement.

This figure plots investors' expected stock position (net of supply shock) $\overline{\left|X_{t}^{i}\right|}$ over time when there is a public announcement. The parameters are set at the following values: $T=50, \lambda=2.0, a_{\theta}=0.85, \sigma_{\Pi}^{2}=0.35, \sigma_{\theta}^{2}=0.1, \sigma_{\delta}^{2}=0.25, \sigma_{S, 1}^{2}=2.0, \sigma_{S, t}^{2}=10^{6}$ $(1<t \leq T-1), \sigma_{Y, t_{A}}^{2}=6.0, \sigma_{Y, t}^{2}=10^{6}\left(t \neq t_{A}\right)$. The announcement dates are, respectively, $t_{A}=8$ and $t_{A}=30$.

Figure 7. Coefficient of the price function $p_{\theta, t}$ when there is a public announcement.

This figure plots the time path of $p_{\theta, t}$, the coefficient of total stock supply $\Theta_{t}$ in the price function, when there is a public announcement. The parameters are set at the following values: $T=50, \lambda=2.0, a_{\Theta}=0.85, \sigma_{\Pi}^{2}=0.35, \sigma_{\Theta}^{2}=0.1, \sigma_{\delta}^{2}=0.25, \sigma_{S, 1}^{2}=2.0$, $\sigma_{S, t}^{2}=10^{6}(1<t \leq T-1), \sigma_{Y, t_{A}}^{2}=6.0, \sigma_{Y, t}^{2}=10^{6}\left(t \neq t_{A}\right)$. The announcement dates are, respectively, $t_{A}=8$ and $t_{A}=30$.

Figure 8. Coefficient of the price function $p_{\Pi, t}$ when there is a public announcement.

This figure plots the time path of $p_{\Pi_{1}, t}$, the coefficient of underlying stock value $\Pi$ in the price function, when there is a public announcement. The parameters are set at the following values: $T=50, \lambda=2.0, a_{\Theta}=0.85, \sigma_{\Pi}^{2}=0.35, \sigma_{\Theta}^{2}=0.1, \sigma_{\delta}^{2}=0.25, \sigma_{S, 1}^{2}=2.0$, $\sigma_{S, t}^{2}=10^{6}(1<t \leq T-1), \sigma_{Y, t_{A}}^{2}=6.0, \sigma_{Y, t}^{2}=10^{6}\left(t \neq t_{A}\right)$. The announcement dates are, 
respectively, $t_{A}=8$ and $t_{A}=30$.

Figure 9. Remaining uncertainty in the underlying value of the stock $\Pi$ when there is a public announcement.

This figure plots $o_{t}^{c}$, the remaining uncertainty in the underlying value of the stock conditional on investors' information, over time when there is a public announcement. The parameters are set at the following values: $T=50, \lambda=2.0, a_{\theta}=0.85, \sigma_{\Pi}^{2}=0.35$, $\sigma_{\Theta}^{2}=0.1, \sigma_{\delta}^{2}=0.25, \sigma_{S, 1}^{2}=2.0, \sigma_{S, t}^{2}=10^{6}(1<t \leq T-1), \sigma_{Y, t_{A}}^{2}=6.0, \sigma_{Y, t}^{2}=10^{6}$ $\left(t \neq t_{A}\right)$. The announcement dates are, respectively, $t_{A}=8$ and $t_{A}=30$.

Figure 10. Volatility of price changes when there is a public announcement.

This figure plots the unconditional volatility of price changes $\operatorname{Var}\left[P_{t}-P_{t-1}\right]$, over time when there is a public announcement. The parameters are set at the following values: $T=50, \lambda=2.0, a_{\Theta}=0.85, \sigma_{n}^{2}=0.35, \sigma_{\Theta}^{2}=0.1, \sigma_{\delta}^{2}=0.25, \sigma_{S, 1}^{2}=2.0, \sigma_{S, t}^{2}=10^{6}$ $(1<t \leq T-1), \sigma_{Y, t_{A}}^{2}=6.0, \sigma_{Y, t}^{2}=10^{6}\left(t \neq t_{A}\right)$. The announcement dates are, respectively, $t_{A}=8$ and $t_{A}=30$. 